\title{
Determinantes de la situación laboral en España: trabajar a tiempo parcial frente a otras situaciones laborales*
}

\author{
Pablo de Pedraza ${ }^{a}$
}

Universidad de Salamanca

\section{Rafael Muñoz de Bustillob}

Universidad de Salamanca

\section{Alberto Villacampa ${ }^{c}$}

Universidad de Salamanca

\section{RESUMEN}

El trabajo estudia los factores explicativos del trabajo a tiempo parcial en el mercado laboral español. Utilizando una muestra de trabajadores, obtenida a partir de la

* Este articulo ha sido realizado gracias a la financiación del Ministerio de Trabajo e Inmigración a través de una subvención del Fondo de Investigación de la Protección Social (proyecto FIPROS 2008/66,

a Pablo de Pedraza. Dpto. de Economía Aplicada. Campus Miguel de Unamuno. Facultad de Derecho 137. Salamanca 37007. Teléfono:+34 923294500 ext.1623. Fax:+34 9232945 16. pablodepedraza@usal.es.

b Rafael Muñoz de Bustillo. Dpto. de Economía Aplicada. Campus Miguel de Unamuno. Facultad de Derecho 147. Salamanca 37007. Telfono: +34 923294500 ext.1689. Fax: :+34 92329 45 16. bustillo@usal.es

c Alberto Villacampa. Dpto. de Economía Aplicada. Campus Miguel de Unamuno. Facultad de Derecho 113. Salamanca 37007. Teléfono: :+34 923294500 ext.1688. Fax: :+34 9232945 16. albervillacampa@hotmail.com

Recibido: Diciembre de 2008 / Aceptado: Junio de 2010. 
Muestra Continua de Vidas Laborales (MCVL 2007), que han tenido alguna relación con la seguridad social a lo largo del 2007, se estudian los determinantes de finalizar el año trabajando a tiempo parcial, con contrato permanente o temporal, en comparación con los determinantes de otras situaciones. Para ello se han identificado y definido las distintas situaciones laborales en las que un trabajador puede finalizar el periodo de muestreo y, utilizando regresiones logísticas, se han estimado los determinantes de las probabilidades de encontrarse en una u otra situación. Las estimaciones se han realizado tanto para el conjunto de la muestra como para hombres y mujeres por separado. Las variables explicativas que se han utilizado se refieren a características del pasado laboral de cada individuo como los tipos de contratos bajo los que ha trabajado, características de la última relación laboral como la categoría profesional y el sector de actividad, variables demográficas como la edad y variables relacionadas con el contexto económico y social como vivir en una Comunidad Autónoma con un PIB superior a la media española. El trabajo concluye que la naturaleza del trabajo a tiempo parcial, aunque guarda algunas similitudes, es muy distinta para hombres y mujeres.

Palabras clave: Mercado de trabajo, trabajo a tiempo parcial, trabajo a tiempo completo, contratos temporales y contratos indefinidos.

Clasificación JEL: J21, J63, J00

\section{AbSTRACT}

This paper studies the determinants of part time employment in the Spanish labour market. Using a sample of workers taken from the administrative data set Muestra Continua de Vidas Laborales (MCVL), we investigate the determinants of employees' finishing 2007 working part time, under a temporary or permanent contract, in comparison with other labour situations. For that purpose, we define a set of possible labour situations and estimate the determinants of each one using logistic regressions. Estimations were carried out for the whole sample and separately for men and women. The explanatoryvariables introduced in the model refer to former work experiences, such as the types of contracts that each individual has had; last job position, such as professional group and sector of activity; demographic characteristics, such as age; and variables related to the economic context, such as living in a region with a GDP above the Spanish national mean. The paper concludes that part time job determinants differ considerably for women and men, although they have several characteristics in common. 
Keywords: labour market, part time job, full time job, temporary contracts, permanent contracts

JEL Classification: J21, J63, J00

\section{INTRODUCCION}

La proporción de población ocupada trabajando a tiempo parcial en España siempre ha sido baja. Mientras en España sólo alrededor del $12 \%$ de los trabajadores están contratados a tiempo parcial, la media de la UE (15) se sitúa en el $21 \%$. Aunque la importancia del trabajo a TP ha aumentado en las últimas décadas, en los años ochenta sólo suponía el $5 \%$, todavía está lejos de la media europea y, por supuesto, del $47 \%$ del caso ciertamente atípico de los Países Bajos.

En este contexto de lenta «normalización» y crecimiento del trabajo a TP, nuestro objetivo es estudiar los determinantes de que un trabajador ocupe un puesto de trabajo a tiempo parcial con un contrato indefinido o con un contrato temporal y en que medida las experiencias laborales pasadas determinan la situación laboral actual. Con ese objetivo, comparamos los determinantes de que un trabajador por cuenta ajena trabaje a tiempo parcial con los determinantes de otras situaciones laborales. Con esa finalidad se ha obtenido una muestra de trabajadores a partir de la Muestra Continua de Vidas Laborales (MCVL) y buscado información sobre el pasado laboral de cada trabajador. El trabajo pretende así desentrañar en el papel que juegan distintas variables referidas al pasado laboral de cada trabajador, como los tipos de contratos con los que se ha trabajado anteriormente, las experiencias de paro o inactividad de larga duración o el número de contratos que se ha firmado a lo largo de la vida, en la situación actual. En este sentido, los resultados aunque no son estrictamente comparables con la literatura de transiciones laborales — del tiempo completo (TC) al tiempo parcial (TP), del TP al TC y del desempleo al TP y/o al TC incluyendo distintos tipos de contratos a TC como a TP - porque no se utiliza únicamente información de la situación laboral inmediatamente anterior a la situación actual sino de todos los estados de los que se ha pasado a lo largo de toda la vida laboral, aportan conclusiones nuevas. Gracias a esta especificación, podemos preguntamos por ejemplo, si los contratos a TP son una vía de entrada al mercado laboral para los jóvenes, si son la antesala del desempleo y/o la jubilación, si los contratos a TP son una herramienta de vuelta al mercado de trabajo para aquellos que en algún momento han interrumpido su carrera durante largos periodos de tiempo y si alguno de estos fenómenos es más característico de los hombres o de las mujeres. En los cálculos también se considera el efecto de otras variables relacionadas con el 
mercado de trabajo como el nivel de ocupación y el sector de actividad, de variables demográficas como la edad y la condición de inmigrante y de variables relacionadas con el contexto económico como vivir en una comunidad autónoma con un PIB per cápita superior al de la media española.

El objetivo que se plantea exige el seguimiento de trabajadores a lo largo del tiempo, algo que sólo se puede hacer parcialmente con las estadísticas laborales más utilizadas, como la Encuesta de Población Activa, diseñadas con la finalidad principal de ofrecer una foto fija, aunque detallada, de la situación del mercado de trabajo en un momento determinado del tiempo.

El trabajo se presenta con la siguiente estructura. En primer lugar, el apartado 2, revisa la literatura más relevante que ha tratado esta cuestión en España. En el apartado 3 se describen las características de la muestra extraída de la Muestra Continua de Vidas Laborales MCVL 2007 que consiste en aquellas personas que tuvieron alguna relación con la seguridad social a lo largo de ese año y que finalizaron el periodo de muestreo en alguna de las siguientes situaciones laborales: trabajando a tiempo completo con un contrato indefinido, trabajando a tiempo completo con un contrato temporal, trabajando a tiempo parcial con un contrato indefinido, trabajando a tiempo parcial con un contrato temporal, cobrando una prestación por desempleo y sin ningún tipo de relación con la seguridad social. A continuación, el apartado 4 ofrece los resultados de las estimaciones de los determinantes de la probabilidad de encontrarse en una u otra situación laboral. Por último, en el apartado 5 se presentan las principales conclusiones alcanzadas y se plantean líneas de investigación futura.

\section{ANTECEDENTES}

El interés por conocer, más allá la situación estática de los distintos colectivos en el mercado de trabajo, sus transiciones laborales, se ha reflejado en la aparición en la última década de toda una serie de estudios sobre los determinantes de dichas transiciones. Así, por ejemplo, y dentro del tema que nos ocupa, Buddelmeyer, Mourre y Ward-Warmedinger (2005) analizan las transiciones entre el trabajo a TP y otros estados laborales para 11 países europeos a partir de la explotación de los datos del Panel de Hogares de la Unión Europea (PHOGUE) entre los años 1994 y 1999. Dicho trabajo obtiene las siguientes conclusiones:

1. El porcentaje de mujeres que estando ocupadas pasan a estar desempleadas no es muy distinto según trabajen a TP o a TC. Por contra el paso a la inactividad 
es significativamente más frecuente desde el trabajo a TP, que desde el trabajo a TC;

2. la permeabilidad entre TP y TC, siendo baja en ambas direcciones, es notablemente mayor del TP al TC;

3. el porcentaje de mujeres que pasan del desempleo al trabajo a TP es menor que el correspondiente a las que pasan a trabajar a TC. Las transiciones de la inactividad al trabajo son muy similares para TP y TC. En este sentido, no parece que el trabajo a TP actúe como un escalón que facilite la entrada en el mercado laboral de las mujeres;

En esta misma línea, un trabajo realizado por la OCDE en 2002 utiliza las cinco primeras olas del PHOGUE, para los países europeos y distintas encuestas longitudinales para Canadá y EE.UU. Algunos de los resultados obtenidos son coherentes con los comentados más arriba, por ejemplo, las transiciones de TP a TC más comunes que las de TC a TP.

El Consejo Económico y Social (CES) realizó en 1996 un informe sobre el trabajo a TP en España utilizando la Encuesta de Población Activa que permite hacer un seguimiento a las unidades domésticas encuestadas durante seis trimestres consecutivos. Los resultados obtenidos confirman, en general, la radiografía presentada más arriba a partir de los datos del PHOGUE. Además, este trabajo realiza un análisis de los factores que afectan a la probabilidad de las personas que trabajan a TP de convertirse en trabajadores a TC frente a mantener su condición de trabajo a TP o pasar a la inactividad, bien por abandono del mercado de trabajo, bien por pasar a ser desempleado. La probabilidad de transición al trabajo a TC es mayor para los hombres, para los trabajadores universitarios (diferencias que desaparecen cuando se analiza sólo a las mujeres) y crece con la antigüedad en el empleo. Por otro lado, la probabilidad es menor para los mayores de 45 años, los trabajadores sin estudios y para los trabajadores del sector «otros servicios» ${ }^{1}$.

Por último, Smith et al. (2000), estudia el periodo inmediatamente posterior al estudiado en el informe del CES y realiza un análisis comparativo de la dinámica de transición en España y el Reino Unido utilizando las respectivas Encuestas de Población Activa que permiten hacer un seguimiento de parte de los trabajadores durante seis cuatrimestres. Entre sus conclusiones destacan las siguientes:

1 Para más detalles véase CES (1996), págs. 74-76. 
1. La mayor parte del trabajo a TP en nuestro país lo ocupan personas con poca capacidad de elección en el mercado de trabajo,

2. las probabilidades de salida del trabajo a TP al trabajo a TC son mayores en España para los hombres y el colectivo más joven, de forma que, con el paso de los años la probabilidad de salida disminuye. Por el contrario, la antigüedad en la empresa disminuye la probabilidad de transición a un trabajo a TC,

3. de las variables ficticias regionales sólo la correspondiente a la región Sur, y en menor medida Centro, muestra un efecto positivo en la probabilidad de salida al TC,

4. en lo que se refiere a las probabilidades de entrada en el TP desde el desempleo o la inactividad, como era de esperar, el ser hombre tiene un fuerte efecto negativo, al igual que ser cabeza de familia o tener bajo nivel educativo. Por contra, ser joven, estudiante o estar buscando un trabajo (ya sea a TP o cualquier trabajo), tienen un efecto positivo sobre la probabilidad de transición del no empleo al empleo a TP.

En conjunto, el análisis de los estudios sobre transiciones laborales de los trabajadores y trabajadoras a TP pone de manifiesto la relativa baja estabilidad de este tipo de trabajo. Este resultado es coherente con el alto grado de involuntariedad detectado en el trabajo a TP en España, ya que es de esperar que los trabajadores involuntarios a TP aprovechen cualquier oportunidad que les ofrezca el mercado de trabajo para cambiar el trabajo por uno de jornada completa. Con todo, los indicadores de transición muestran que un porcentaje muy significativo de trabajadores a TP, entre el 30 y el $60 \%$ dependiendo del período de análisis, el sexo y la fuente consultada, que mantienen su condición de trabajadores a TP a lo largo del tiempo, lo que podría reflejar la aparición de un grupo estable de trabajadores a TP en nuestro país.

\section{Características de la muestra de trabajadores OBTENIDA DE LA MCVL 2007}

La Muestra Continua de Vidas Laborales 2007, MCVL, es un conjunto organizado de microdatos anónimos extraídos de registros administrativos de la Seguridad Social complementados, en su caso, con datos del Padrón Municipal Continuo y datos fiscales de la Agencia Tributaria. Incluyen algo más de un millón de personas (un $4 \%$ de la población de referencia) y constituyen una muestra representativa de todas las personas que tuvieron relación con la Seguridad Social en un determinado año, en este caso en 2007. La muestra se actualiza anualmente y recoge información sobre la 
población cotizante o pensionista en el año de referencia, incluyendo su historial para las variables procedentes de la Seguridad Social, remontándose hacia atrás hasta donde se conserven registros informatizados ${ }^{2}$.

Partiendo de la MCVL 2007 se ha extraído una submuestra de 559,453 individuos que tuvieron alguna relación con la seguridad social a lo largo de 2007. A pesar de que la MCVL cuenta con más de un millón de individuos, la muestra con la que estamos trabajando tiene un tamaño considerablemente menor porque, con el doble objetivo de hacer un análisis más riguroso y de facilitar la interpretación de los resultados, hemos aplicado una serie de filtros. Se ha eliminado de la muestra, y por tanto del análisis, a los trabajadores que terminaron el periodo de muestreo como autónomos y a los fijos discontinuos. Las peculiaridades de ambos tipos de trabajadores hacen recomendable su exclusión. Por lo tanto el trabajo se centra en los trabajadores por cuenta ajena que no son fijos discontinuos ${ }^{3}$ sin perjuicio de que hayan trabajado alguna vez por cuenta propia. También se ha eliminado del análisis a aquellos trabajadores de los que se desconocía su ocupación (en su mayoría eran autónomos) y a aquellas observaciones en las que no se podía identificar si el trabajador era inmigrante económico o no. Todos los individuos de la muestra han sido clasificados según su situación laboral a cierre de muestra (31 de diciembre de 2007) de la siguiente manera:

- Trabajando a tiempo completo con un contrato indefinido;

- Trabajando a tiempo completo con un contrato temporal;

${ }^{2}$ La principal diferencia entre la EPA y la MCVL es la no inclusión en ésta de los trabajadores con un sistema de previsión social distinto a la Seguridad Social (en particular los funcionarios de Clases Pasivas), o los que no tienen ninguno (empleo sumergido y algunas actividades marginales que, al menos en principio, sí estarían recogidas en la EPA). Así mismo, la población definida para la MCVL no se corresponde conceptualmente con la población activa, sino que se asemeja más a la de «perceptores de ingresos» que aparece en las tablas de hogares de la EPA. Aunque los ámbitos poblacionales y temporales sean diferentes, es posible extraer de ambas fuentes grupos equiparables (Ramos Muñoz, 2007). Una descripción de la MCVL se puede encontrar en Durán y Sevilla (2005) y en la web de la SS (http://wwwl.seg-social.es/stpri01/idcplg?IdcService $=S S_{-}$ GET_PAGE\&nodeId $=211 \& E S T I L O=1)$. Una descripción detallada de cómo se ha obtenido la extracción con la que se realizan estas estimaciones se puede encontrar en el informe www.segsocial.es/Internet_1/Estadistica/FondodeInvestigacio48073/EstudiosFIPROS/FIPROS2008/index. htm\#documentoPDF.

${ }^{3}$ De acuerdo con la EPA, en 2007 los trabajadores asalariados con contrato fijo discontinuo suponían el $1,4 \%$ del total de asalariados 
- Trabajando a tiempo parcial con un contrato indefinido;

- Trabajando tiempo parcial con un contrato temporal;

- Cobrando prestación desempleo;

- Sin cobrar prestación desempleo.

Es necesario matizar que ninguna de las dos últimas situaciones se corresponde con las definiciones habituales de desempleo e inactividad. La primera de ellas, cobrando prestación por desempleo, no puede ser considerada representativa de los desempleados a cierre de muestra pues no incluye los desempleados que no estén cobrando prestación. La segunda, sin cobrar prestación por desempleo, no puede ser considerada representativa de las personas inactivas pues no incluye a los inactivos que no hayan tenido una relación con la Seguridad Social a lo largo de 2007. En ambos casos se trata de personas que, después de haber tenido algún tipo de relación con la SS durante 2007, finalizan el muestreo sin trabajar. Este colectivo se denominará de aquí en adelante personas que terminan el muestreo sin trabajar ${ }^{4}$.

TABLA 1.-Composición por situaciones laborales a cierre de muestra y porcentaje de hombres y mujeres en cada situación laboral

\begin{tabular}{|c|c|c|c|c|}
\hline & \multicolumn{2}{|c|}{ Frecuencias absolutas y relativas } & $\begin{array}{c}\text { Porcentaje de } \\
\text { Hombres en } \\
\text { cada situación }\end{array}$ & $\begin{array}{c}\text { Porcentaje de } \\
\text { Mujeres en cada } \\
\text { situación }\end{array}$ \\
\hline $\begin{array}{c}\text { Tiempo } \\
\text { completo } \\
\text { indefinido }\end{array}$ & 266,635 & $47.66 \%$ & $62.49 \%$ & $37.51 \%$ \\
\hline
\end{tabular}

${ }^{4}$ Deliberadamente no hemos denominado parados a los que cobran una prestación por desempleo ni inactivos a los que dejan de aparecer en la muestra porque no tienen ningún tipo de relación con la seguridad social. El grupo de aquellos que cobran una prestación por desempleo no coincide con el lo que habitualmente consideramos desempleados. Dentro de los desempleados estarían también las personas que buscan trabajo, aunque no tenga derecho a recibir una prestación por desempleo. Este sería el caso de los jóvenes que buscan su primer trabajo. Por el mismo motivo, aquellos que pasan a no tener ningún tipo de relación con la seguridad social no coinciden exactamente con los inactivos que han abandonado la población activa. Puede que se encuentren buscando trabajo y por tanto, sean en realidad parados. Este sería el caso de aquellos que han agotado su prestación por desempleo antes de ocupar un puesto de trabajo. 
TABLA 1 (cont.).-Composición por situaciones laborales a cierre de muestra y porcentaje de hombres y mujeres en cada situación laboral

\begin{tabular}{|c|c|c|c|c|}
\hline & \multicolumn{2}{|c|}{ Frecuencias absolutas y relativas } & $\begin{array}{c}\text { Porcentaje de } \\
\text { Hombres en } \\
\text { cada situación }\end{array}$ & $\begin{array}{c}\text { Porcentaje de } \\
\text { Mujeres en cada } \\
\text { situación }\end{array}$ \\
\hline $\begin{array}{c}\text { Tiempo } \\
\text { completo } \\
\text { temporal }\end{array}$ & 110,437 & $19.74 \%$ & $65.99 \%$ & $34.01 \%$ \\
\hline $\begin{array}{c}\text { Tiempo parcial } \\
\text { indefinido }\end{array}$ & 34,993 & $6.25 \%$ & $25.06 \%$ & $74.94 \%$ \\
\hline $\begin{array}{c}\text { Tiempo parcial } \\
\text { temporal }\end{array}$ & 31,176 & $5.57 \%$ & $31.28 \%$ & $68.72 \%$ \\
\hline $\begin{array}{c}\text { Cobrando } \\
\text { prestación } \\
\text { desempleo }\end{array}$ & 53,705 & $9.60 \%$ & $44.98 \%$ & $55.02 \%$ \\
\hline $\begin{array}{c}\text { Sin cobrar } \\
\text { prestación } \\
\text { desempleo }\end{array}$ & 62,507 & $11.17 \%$ & $49.48 \%$ & $50.52 \%$ \\
\hline Total & 559,453 & $100.0 \%$ & $\begin{array}{c}313,106 \\
(55.97 \%)\end{array}$ & $\begin{array}{c}246,347 \\
(44.03 \%)\end{array}$ \\
\hline
\end{tabular}

Fuente: Elaboración propia a partir de la MCVL 2007.

La tabla 1 recoge el número y el porcentaje de individuos de la muestra que se encuentran en cada situación laboral y la distribución de cada situación por sexos. Las dos situaciones más comunes son trabajar a tiempo completo con un contrato indefinido y trabajar a tiempo completo con un contrato temporal. En ambos casos la mayoría, más del $60 \%$, son hombres mientras que en el resto de situaciones, trabajar a tiempo parcial, con contrato indefinido o temporal, y no trabajar al final del muestreo, predominan las mujeres.

La tabla 2 recoge los porcentajes de trabajadores que se encuentran en cada una de las categorías de las variables cuyo efecto en la situación laboral a cierre de muestra es objeto de estudio, tanto en el conjunto de la muestra como en cada situación laboral. La primera línea recoge el porcentaje de mujeres, que suponen algo más del $44 \%$ de la muestra pero solamente entre el 34 y el $37 \%$ de los trabajadores a tiempo completo. En contraste, las mujeres están sobrerepresentadas entre los que finalizan el periodo sin trabajar, entre los que el 50-55\% son mujeres, y entre los que trabajan a tiempo parcial, entre los que el 68-75\% son mujeres.

En cuanto a la distribución por sectores observamos que la estructura del conjunto de la muestra es muy similar a lo que podemos esperar de la población activa en Espa- 
ña. Tomando como referencia los porcentajes del conjunto de la muestra, la proporción de agricultores aumenta tanto entre los que trabajan a tiempo completo con un contrato temporal como entre los que terminan el periodo de muestreo sin trabajar, sobre todo entre los que terminan cobrando la prestación por desempleo; la proporción de trabajadores de la industria es mayor entre los que trabajan a tiempo completo con un contrato indefinido mientras que la de los trabajadores de la construcción aumenta entre los que trabajan a tiempo completo con un contrato temporal; la proporción de trabajadores del comercio con un contrato indefinido bien a tiempo completo bien a tiempo parcial es mayor que su peso en el empleo; en contraste, el trabajo a tiempo parcial, ya sea con un contrato indefinido o con un contrato temporal, es claramente superior a su peso en el empleo en el sector servicios.

En lo que se refiere a los niveles de cualificación ${ }^{5}$, y siguiendo con la comparación entre el conjunto de la muestra y cada situación laboral observamos lo siguiente. El porcentaje de trabajadores con un nivel de cualificación alto aumenta entre los que trabajan a tiempo completo indefinidos y entre los que trabajan a tiempo parcial temporalmente. El porcentaje de trabajadores con un nivel de cualificación medio se mantiene bastante estable en casi todos los grupos aunque disminuye entre los que trabajan a tiempo parcial, sobre todo entre los que lo hacen con un contrato temporal. El porcentaje de trabajadores con un nivel de cualificación bajo disminuye entre los que trabajan a tiempo completo con un contrato indefinido y aumenta en las demás situaciones laborales.

Los cuatro variables siguientes recogen información sobre el pasado laboral. En primer lugar, la variable haber cambiado de sector a lo largo de la vida laboral (cambio s.) refleja que en algún momento de su vida laboral esa persona ha trabajado en algún sector distinto de aquel en el que trabaja actualmente. Para generar esta variable se ha utilizado la clasificación de sectores anterior: agricultura, industria, construcción, comercio y servicios. En el caso de aquellos que finalizan el periodo sin trabajar se ha tomado como referencia la última relación laboral. Casi el 70\% de la muestra había trabajado alguna vez en algún sector distinto a aquel en el que lo hacía el 31 de diciembre de 2007. Como era de esperar, este porcentaje aumenta entre aquellos que trabajan a tiempo completo con un contrato temporal y entre los que termina el muestreo cobrando una prestación por desempleo.

${ }^{5}$ Los tres niveles de cualificación (bajo, medio y alto) se han generado a partir del al variable de la MCVL que recoge el grupo de cotización. La MCVL no tienen información sobre los niveles educativos. 
En segundo lugar, la variable número de contratos (contratos) recoge el número de contratos que cada persona ha firmado a lo largo de de su vida laboral, esta variable se ha organizado en los intervalos que aparecen en la tabla. Más del 75\% de las personas que componen la muestra ha firmado entre 3 y 20 y muchos, casi un $30 \%$, entre 6 y 10 contratos. Las proporciones son muy parecidas para aquellos con contrato indefinido, tanto a tiempo completo como a tiempo parcial, que en ambos casos, en general, habrían tenido menos relaciones laborales que el conjunto de la muestra. Lo contrario ocurre con los trabajadores con contrato temporal, tanto a tiempo completo como a tiempo parcial, entre los que, como era de esperar, aumentan claramente las proporciones de los intervalos más altos. Además, entre aquellos que finalizan el muestreo sin trabajar, también se percibe una ligera acumulación en los intervalos más altos. Estos resultados pueden considerarse como una evidencia de la existencia de un proceso de atrapamiento en la temporalidad en el mercado laboral español.

TABLA 2.-Frecuencias relativas del conjunto de la muestra y de cada situación laboral por sexo, sector, ocupación, pasado laboral, edad y contexto económico

\begin{tabular}{|c|c|c|c|c|c|c|c|}
\hline & \multirow{2}{*}{$\begin{array}{c}\text { Porcentajes } \\
\text { muestra }\end{array}$} & \multicolumn{2}{|c|}{ A tiempo completo } & \multicolumn{2}{|c|}{ A tiempo parcial } & \multicolumn{2}{|c|}{ Sin un puesto de trabajo } \\
\hline & & $\begin{array}{l}\text { Contrato } \\
\text { indefinido }\end{array}$ & $\begin{array}{l}\text { Contrato } \\
\text { temporal }\end{array}$ & $\begin{array}{l}\text { Contrato } \\
\text { indefinido }\end{array}$ & $\begin{array}{l}\text { Contrato } \\
\text { temporal }\end{array}$ & $\begin{array}{l}\text { Cobrando } \\
\text { prestación }\end{array}$ & Sin cobrar \\
\hline Mujeres & 44.03 & 37.51 & 34.01 & 74.94 & 68.72 & 55.02 & 50.52 \\
\hline \multicolumn{8}{|l|}{ Sector } \\
\hline agricultura & 1.92 & 0.97 & 2.68 & 0.3 & 0.23 & 6.58 & 2.42 \\
\hline industria & 15.48 & 21.88 & 11.24 & 6.64 & 7.1 & 12.91 & 7.01 \\
\hline construcción & 13.22 & 7.9 & 29.51 & 2.59 & 2.49 & 15.42 & 16.58 \\
\hline comercio & 17.63 & 21.77 & 9.61 & 28.56 & 11.04 & 15.99 & 12.72 \\
\hline servicios & 51.75 & 47.48 & 46.96 & 61.91 & 79.14 & 49.1 & 61.27 \\
\hline \multicolumn{8}{|l|}{ Ocupación } \\
\hline Alto & 16.51 & 21.66 & 13.21 & 11.22 & 16.7 & 5.43 & 12.78 \\
\hline Medio & 44.19 & 47.01 & 45.71 & 36.8 & 27.49 & 46.03 & 40.41 \\
\hline Bajo & 39.29 & 31.33 & 41.07 & 51.98 & 55.82 & 48.54 & 46.81 \\
\hline Cambio $S$. & 69.63 & 66.63 & 77.69 & 61.77 & 66.35 & 77.68 & 67.28 \\
\hline \multicolumn{8}{|l|}{ Contratos } \\
\hline 1 & 3.03 & 4.48 & 0.49 & 2.88 & 1.65 & 2.03 & 2.91 \\
\hline 2 & 6.4 & 8.89 & 2.72 & 8.52 & 3.3 & 5.45 & 3.45 \\
\hline $3-5$ & 24.75 & - & - & - & - & - & - \\
\hline
\end{tabular}


TABLA 2 (cont.).-Frecuencias relativas del conjunto de la muestra y de cada situación laboral por sexo, sector, ocupación, pasado laboral, edad y contexto económico

\begin{tabular}{|c|c|c|c|c|c|c|c|}
\hline & \multirow{2}{*}{$\begin{array}{c}\text { Porcentajes } \\
\text { muestra }\end{array}$} & \multicolumn{2}{|c|}{ A tiempo completo } & \multicolumn{2}{|c|}{ A tiempo parcial } & \multicolumn{2}{|c|}{ Sin un puesto de trabajo } \\
\hline & & $\begin{array}{l}\text { Contrato } \\
\text { indefinido }\end{array}$ & $\begin{array}{l}\text { Contrato } \\
\text { temporal }\end{array}$ & $\begin{array}{c}\text { Contrato } \\
\text { indefinido }\end{array}$ & $\begin{array}{l}\text { Contrato } \\
\text { temporal }\end{array}$ & $\begin{array}{l}\text { Cobrando } \\
\text { prestación }\end{array}$ & Sin cobrar \\
\hline $6-10$ & 29.16 & 29.78 & 26.33 & 30.09 & 35.89 & 24.7 & 31.49 \\
\hline $11-20$ & 23.78 & 19.59 & 30.92 & 23.97 & 26.04 & 27.7 & 24.41 \\
\hline $21-30$ & 7.21 & 4.96 & 10.77 & 5.04 & 6.14 & 11.88 & 8.22 \\
\hline $\begin{array}{l}\text { Más de } 30 \\
>365\end{array}$ & 5.61 & 2.78 & 9.69 & 3.56 & 4.65 & 10.14 & 8.24 \\
\hline Úlimos 5a. & 25.46 & 14.2 & 30.95 & 23.54 & 39.55 & 41.92 & 43.65 \\
\hline Anterior & 38.06 & 38.7 & 37.11 & 39.00 & 27.09 & 49.45 & 32.13 \\
\hline \multicolumn{8}{|l|}{$\begin{array}{l}\text { Contratos } \\
\text { anteriores }\end{array}$} \\
\hline fijos_tp & 11.61 & 7.55 & 9.37 & 34.01 & 15.1 & 17.39 & 13.62 \\
\hline temp_tc & 70.65 & 57.19 & 93.41 & 46.42 & 63.25 & 84.57 & 85.02 \\
\hline temp_tp & 47.92 & 36.65 & 50.15 & 66.62 & 81.53 & 52.82 & 60.63 \\
\hline fijos_tc & 43.15 & 50.27 & 33.21 & 38.39 & 27 & 42.33 & 41.72 \\
\hline autónomo & 13.17 & 12.52 & 13.63 & 11.5 & 16.16 & 14.99 & 13.02 \\
\hline \multicolumn{8}{|l|}{ Edad } \\
\hline$<20$ & 0.57 & 0.18 & 0.89 & 0.46 & 0.82 & 0.28 & 1.86 \\
\hline $21-25$ & 8.68 & 5.2 & 11.48 & 10.28 & 10.36 & 7.41 & 17.96 \\
\hline $26-30$ & 16.61 & 14.26 & 22.42 & 14.9 & 18.5 & 16.88 & 16.14 \\
\hline $31-35$ & 17.12 & 17.63 & 19.03 & 16.35 & 20 & 13.47 & 14.07 \\
\hline $36-40$ & 16.3 & 17.46 & 15.28 & 19.24 & 14.72 & 15.61 & 12.92 \\
\hline $41-45$ & 13.48 & 14.44 & 12.79 & 15.52 & 7.2 & 9.29 & 16.22 \\
\hline $46-50$ & 10.12 & 12.03 & 7.53 & 9.33 & 8.77 & 9.75 & 8.03 \\
\hline $51-55$ & 7.86 & 9.1 & 6.64 & 6.51 & 6.69 & 9.53 & 4.6 \\
\hline $56-60$ & 5.38 & 6.65 & 2.81 & 4.42 & 2.34 & 9.62 & 2.96 \\
\hline $61-65$ & 3.29 & 2.81 & 1.33 & 2.25 & 10.25 & 7.45 & 2.34 \\
\hline+ de 65 & 0.59 & 0.24 & 0.1 & 0.74 & 0.35 & 0.71 & 2.90 \\
\hline Emigrante & 15.91 & 10.44 & 20.1 & 15.59 & 28.48 & 17.68 & 24.26 \\
\hline
\end{tabular}




\begin{tabular}{|l|c|c|c|c|c|c|c|}
\hline & \multirow{2}{*}{$\begin{array}{c}\text { Porcentajes } \\
\text { muestra }\end{array}$} & \multicolumn{2}{|c|}{ A tiempo completo } & \multicolumn{2}{c|}{ A tiempo parcial } & \multicolumn{2}{c|}{ Sin un puesto de trabajo } \\
\cline { 3 - 8 } & $\begin{array}{c}\text { Contrato } \\
\text { indefinido }\end{array}$ & $\begin{array}{c}\text { Contrato } \\
\text { temporal }\end{array}$ & $\begin{array}{c}\text { Contrato } \\
\text { indefinido }\end{array}$ & $\begin{array}{c}\text { Contrato } \\
\text { temporal }\end{array}$ & $\begin{array}{c}\text { Cobrando } \\
\text { prestación }\end{array}$ & Sin cobrar \\
\hline Contexto & & & & & & & \\
PIB & 50.36 & 54.1 & 42.9 & 50.42 & 57.99 & 42.57 & 50.41 \\
$>40000$ & 59.86 & 61.74 & 57.01 & 61.49 & 69.91 & 51.79 & 57.9 \\
Provincia $\neq$ & 35.5 & 32.29 & 35.19 & 34.2 & 47.62 & 40.78 & 39.85 \\
\hline Núm. Obs. & 559,453 & 266,635 & 110,437 & 34,993 & 31,176 & 53,705 & 62,507 \\
\hline
\end{tabular}

Fuente: Elaboración propia a partir de la MCVL 2007.

En tercer lugar, las experiencias de desempleo e inactividad ${ }^{6}$ que han durado más de 365 (>365) han sido divididas en dos tipos: aquellas anteriores a los últimos cinco años y aquellas más recientes (que han tenido lugar en los últimos 5 años). Más del $25 \%$ de la población ha tenido una experiencia de este tipo en los últimos 5 años. Esta proporción aumenta entre los que trabajan con contrato temporal, sobre todo entre los que lo hacen a tiempo parcial y entre los que terminan el periodo sin trabajo. Por otra parte, casi el $40 \%$ ha tenido una experiencia de desempleo e inactividad de más de 365 días en algún momento de su vida laboral sin contar los últimos cinco años. Este porcentaje es bastante homogéneo entre los distinto grupos que terminan el periodo de muestreo trabajando con la excepción de aquellos que trabajan temporalmente a tiempo parcial, entre los que dicha proporción desciende al $27 \%$.

En cuarto lugar, se ha identificado a los individuos que alguna vez en su vida laboral ha trabajado bajo alguno de los siguientes tipos de contratos (contratos anteriores) sin contar la situación actual: contrato fijo a tiempo parcial (fijos_tp), contrato temporal a tiempo completo (temp_tc), contrato temporal a tiempo parcial (temp_tp), contrato fijo a tiempo completo (fijo_tc) y haber sido autónomo (autónomo).

Algo más de un $11 \%$ de la muestra ha trabajado alguna vez con un contrato fijo a tiempo parcial. Este porcentaje aumenta claramente, hasta el 34\%, entre los que tienen actualmente este tipo de contrato y disminuye, al $7 \%$, entre los que trabajan con un contrato indefinido a tiempo completo.

${ }^{6}$ Por los criterios de confección de la MCVL, que recoge las relaciones de los individuos con la Seguridad Social, la definición de desempleo dista bastante de la habitual: en la MCVL un desempleado es exclusivamente aquella persona que está cobrando la prestación por desempleo, independientemente de si busca trabajo o no. 
Más del 70\% ha trabajado alguna vez con un contrato temporal a tiempo completo, porcentaje que aumenta, hasta el $93 \%$, entre los que tienen actualmente este tipo de contrato y alrededor del $85 \%$ entre los que finalizan sin trabajar y, por el contrario, desciende entre los que trabajan con carácter indefinido tanto a tiempo parcial como a tiempo completo.

Casi un $48 \%$ han sido alguna vez trabajadores temporales a tiempo parcial, este porcentaje aumenta en todas las situaciones laborales, sobre todo entre los que actualmente están en esta situación, con excepción de aquellos que trabajan a tiempo completo de forma indefinida.

Finalmente, aunque solo algo más del $47 \%$ de la muestra trabaja actualmente con un contrato indefinido a tiempo completo (tabla 1) sólo algo más de un $43 \%$ han trabajado alguna vez, sin contar la actual, con un contrato de este tipo. Dicho porcentaje aumenta al $50 \%$ entre los que actualmente trabajan con un contrato de este tipo, se reduce en el resto de situaciones laborales pero se mantiene más o menos constante entre los que finalizan el periodo sin trabajar. Finalmente, el porcentaje de persona que ha sido alguna vez autónomos se mantiene más o menos constante tanto en el conjunto de la muestra como por situaciones actuales, aunque aumenta ligeramente entre los trabajadores temporales a tiempo parcial y entre los que finalizan el periodo de muestro cobrando la prestación por desempleo.

La anterior comparación entre situaciones actuales y pasadas ofrece, de nuevo, evidencias de la existencia de atrapamiento en determinadas situaciones laborales de temporalidad. Además, el hecho de haber tenido un contrato indefinido no parece implicar estabilidad laboral total en el sentido de no terminar el periodo de muestreo sin trabajar. Llama así enormemente la atención, especialmente dado el cariz que ha tomado el debate sobre la presunta dificultad de despedir en España a los trabajadores con contrato fijo (más bien indefinido), el hecho de que alrededor del $40 \%$ de los «desempleados» ( in puesto de trabajo al cierre de la muestra) hayan tenido un contrato «fijo» en el pasado, siendo el porcentaje similar entre aquellos con y sin derecho a prestaciones. Por último, entre los que han sido autónomos el trabajo temporal a tiempo parcial parece ser algo bastante común.

En cuanto a las frecuencias por intervalos de edad se pueden destacar tres cosas. Primero, los que trabajan con un contrato indefinido, tanto a tiempo completo como a tiempo parcial, son en general mayores que los que trabajan con un contrato temporal. Segundo, terminar el periodo sin trabajar y sin cobrar prestación se concentra en edades más jóvenes que en el caso de recibir prestaciones por desempleo circunstancia que, por otra parte, si bien se concentra ligeramente entre los 25 y los 40 tiene una distribución más homogénea entre los distintos intervalos de edad que el resto de situaciones. 
En último lugar, llama la atención la importancia del trabajo temporal a tiempo parcial previo a la jubilación pues más de un $10 \%$ de los trabajadores temporales a tiempo parcial tiene entre 61 y 65 años cuando las personas de dicha edad sólo suponen algo más del $3 \%$ de la muestra.

Casi un $16 \%$ de la muestra son emigrantes económicos ${ }^{7}$, proporción que aumenta entre los contratados temporalmente y entre los que finalizan el periodo de muestreo sin trabajar y sin cobrar prestación. Aproximadamente la mitad de la muestra trabaja en una de las regiones que tiene un PIB per cápita superior a la media donde la proporción de contratados indefinidamente a tiempo completo es superior, al igual que los que trabajan con un contrato temporal a tiempo parcial. Casi el $60 \%$ vive en una población de más de 40000 habitantes y aproximadamente el $35 \%$ en una provincia distinta a aquella en la que nació.

La tabla 3 amplia el análisis descriptivo ofreciendo las frecuencias relativas de los hombres y mujeres del conjunto de la muestra y las de los hombres y mujeres que finalizan el periodo de muestreo trabajando a tiempo parcial, bien con un contrato indefinido bien con un contrato temporal. Como se señalaba anteriormente el trabajo a tiempo parcial es poco frecuente en España. Además de escaso, el trabajo a tiempo parcial (como ocurre en el resto de la UE) es esencialmente un fenómeno femenino. La incidencia del trabajo a tiempo parcial es mucho mayor entre las mujeres, casi 48,000 mujeres de las 246,347 que componen la muestra terminó el periodo de muestreo trabajando a tiempo parcial, mientras que solo algo más de 18,000 hombres de los más de 300,000 que componen la muestra lo hizo. En general, las mujeres se concentran más en el sector servicios y en el comercio mientras que los hombres lo hacen también en el sector industrial y en el de la construcción. Sin embargo, estas diferencias sectoriales

${ }^{7}$ La categoría emigrantes económicos engloba a nacionales de los siguientes países: países europeos no pertenecientes a la UE-15, Latinoamérica, África y Asia - Pacífico. Son personas provenientes de países con un nivel de desarrollo inferior al español, que vienen a España por motivos laborales, buscando mejorar la situación económica que soportan en sus países de origen. Por motivos de anonimización, el nivel de desagregación de los grupos de países con un menor número de representantes en la MCVL es escaso, por lo que dentro de la categoría de emigrantes económicos se incluye también a los ciudadanos de países desarrollados como Japón, Australia, Nueva Zelanda, El Vaticano, Islandia, Lienchestein, Israel, Mónaco, Noruega, San Marino, Suiza o Andorra, cuya situación, siguiendo el criterio establecido, se asemejaría más a la de inmigrantes no económicos. Sin embargo, dado el escaso volumen de inmigrantes que aportan los países mencionados en relación al del conjunto de la categoría a la que pertenecen, consideramos que la clasificación sigue siendo válida. 
se atenúan al comparar las muestras de hombres y mujeres que finalizan el periodo de muestreo trabajando a tiempo parcial, lo que refleja la importancia de los determinantes de demanda (i.e. de naturaleza sectorial) de este tipo de contrato.

TABLA 3.-Frecuencias relativas del conjunto de la muestra por sexos y frecuencias relativas de los trabajadores a tiempo parcial (con contrato indefinido y temporal) por sexos

\begin{tabular}{|c|c|c|c|c|c|c|}
\hline & \multicolumn{2}{|c|}{$\begin{array}{c}\text { Hombres } \\
\text { A tiempo parcial }\end{array}$} & \multicolumn{2}{|c|}{$\begin{array}{c}\text { Mujeres } \\
\text { A tiempo parcial }\end{array}$} & \multicolumn{2}{|c|}{ Conjunto de la muestra } \\
\hline & Indefinido & Temporal & Indefinido & Temporal & Hombres & Mujeres \\
\hline Sector & & & & & & \\
\hline agricultura & 0.56 & 0.4 & 0.19 & 0.13 & 3.64 & 2.11 \\
\hline industria & 7.75 & 19.17 & 5.87 & 2.35 & 19.21 & 9.98 \\
\hline construcción & 4.34 & 5.76 & 1.83 & 7.36 & 23.27 & 3.22 \\
\hline comercio & 35.38 & 10.29 & 25.07 & 23.15 & 15.45 & 19.04 \\
\hline servicios & 51.97 & 64.39 & 67.05 & 67.01 & 38.42 & 65.65 \\
\hline \multicolumn{7}{|l|}{ Ocupación } \\
\hline Alto & 17.6 & 15.02 & 8.81 & 8.36 & 13.09 & 13.49 \\
\hline Medio & 35.3 & 55.45 & 30.15 & 48.79 & 51.23 & 35.68 \\
\hline Bajo & 47.1 & 29.5 & 61.04 & 42.85 & 35.68 & 50.82 \\
\hline Cambio $S$. & 56.41 & 61.26 & 50.96 & 65.33 & 75.66 & 58.66 \\
\hline \multicolumn{7}{|l|}{ Contratos } \\
\hline 1 & 19.67 & 3.51 & 6.95 & 0.84 & 4.63 & 2.84 \\
\hline 2 & 4.87 & 5.35 & 9.26 & 1.9 & 4.3 & 5.18 \\
\hline $3-5$ & 18.73 & 33.76 & 28.82 & 35.24 & 25.36 & 30.83 \\
\hline $6-10$ & 25.03 & 22.62 & 25.7 & 23.7 & 27.53 & 27.01 \\
\hline $11-20$ & 20.68 & 20.48 & 19.88 & 25.86 & 23.18 & 21.2 \\
\hline $21-30$ & 4.85 & 7.02 & 4.8 & 5.93 & 7.39 & 6.3 \\
\hline Más de 30 & 4.20 & 5.12 & 3.21 & 4.64 & 5.9 & 4.59 \\
\hline \multicolumn{7}{|l|}{$>365$} \\
\hline Últimos 5a. & 20.46 & 21.44 & 20.9 & 39.44 & 14.32 & 23.56 \\
\hline Anterior & 36.03 & 31.96 & 37.49 & 25.99 & 38.4 & 33.54 \\
\hline \multicolumn{7}{|l|}{ Anteriores } \\
\hline fijos_tp & 25 & 9.75 & 31.68 & 12.53 & 6.06 & 14.86 \\
\hline temp_tc & 49.01 & 62.97 & 45.72 & 65.09 & 71.31 & 69.75 \\
\hline
\end{tabular}


TABLA 3 (cont.).-Frecuencias relativas del conjunto de la muestra por sexos y frecuencias relativas de los trabajadores a tiempo parcial (con contrato indefinido y temporal) por sexos

\begin{tabular}{|c|c|c|c|c|c|c|}
\hline & \multicolumn{2}{|c|}{$\begin{array}{c}\text { Hombres } \\
\text { A tiempo parcial }\end{array}$} & \multicolumn{2}{|c|}{$\begin{array}{c}\text { Mujeres } \\
\text { A tiempo parcial }\end{array}$} & \multicolumn{2}{|c|}{ Conjunto de la muestra } \\
\hline & Indefinido & Temporal & Indefinido & Temporal & Hombres & Mujeres \\
\hline temp_tp & 50.83 & 45.85 & 62.78 & 63.36 & 30.58 & 57.28 \\
\hline fijos_tc & 30.12 & 49.39 & 32.92 & 15.88 & 44.97 & 35.4 \\
\hline autónomo & 19.11 & 14.97 & 8.69 & 7.84 & 14.28 & 8.47 \\
\hline \multicolumn{7}{|l|}{ Edad } \\
\hline$<20$ & 0.47 & 0.64 & 0.12 & 0.27 & 0.33 & 0.18 \\
\hline $21-25$ & 9.29 & 10.54 & 5.06 & 20.66 & 7.33 & 9.33 \\
\hline $26-30$ & 13.25 & 11.68 & 19.77 & 24.16 & 14.61 & 21.67 \\
\hline $31-35$ & 13.07 & 25.12 & 20.7 & 16.9 & 19.59 & 19.2 \\
\hline $36-40$ & 12.12 & 6.58 & 16.22 & 8.76 & 14 & 14.02 \\
\hline $41-45$ & 10.18 & 5.67 & 12.27 & 13.95 & 13.56 & 11.47 \\
\hline $46-50$ & 25.59 & 4.42 & 8.85 & 5.85 & 10.58 & 10.08 \\
\hline $51-55$ & 6.10 & 2.96 & 10.82 & 3.63 & 8.8 & 6.67 \\
\hline $56-60$ & 4.6 & 2.92 & 4.0 & 2.12 & 6.46 & 4.77 \\
\hline $61-65$ & 3.17 & 28.77 & 1.85 & 3.46 & 4.03 & 2.18 \\
\hline+ de 65 & 2.16 & 0.69 & 0.32 & 0.24 & 0.69 & 0.44 \\
\hline Emigrante & 23.96 & 20.27 & 23.67 & 42.52 & 23.47 & 24.68 \\
\hline \multicolumn{7}{|l|}{ Contexto } \\
\hline PIB & 41.9 & 51.04 & 49.9 & 30.98 & 40.66 & 45.93 \\
\hline$>40000$ & 50.94 & 65.63 & 62.08 & 54.15 & 53.78 & 56.51 \\
\hline Provincia $\neq$ & 41.0 & 44.17 & 41.17 & 55.36 & 40.66 & 42.4 \\
\hline Núm. Obs. & 8,769 & 9,751 & 26,224 & 21,425 & 313,106 & 246,347 \\
\hline
\end{tabular}

Fuente: Elaboración propia a partir de la MCVL 2007.

En el conjunto de la muestra, alrededor del $13 \%$ de mujeres y hombres desarrollan su actividad en una ocupación de nivel de cualificación alto. Al comparar con el conjunto de hombres y mujeres trabajando a tiempo parcial, observamos que el porcentaje personas con niveles de calificación alta aumenta entre los hombres y disminuye entre las mujeres. En cuanto al número de contratos, las mujeres se concentran en niveles intermedios (entre 3 y 10 contratos) mientras que la distribución de frecuencias del 
número de contratos tiene una forma más plana para los hombres, algo que se sigue observando entre los que trabajan a tiempo parcial.

El porcentaje de hombres que han trabajado alguna vez a tiempo completo, sobre todo el de aquellos que lo han hecho con un contrato fijo (y como autónomos) es mayor que el porcentaje de mujeres. Por el contrario, el porcentaje de mujeres que ha trabajado alguna vez a tiempo parcial es superior al de hombres.

Las frecuencias relativas de mujeres menores de 30 son mayores que las de los hombres. Sin embargo, las frecuencias relativas de hombres mayores de 50 son mayores que las de las mujeres. En edades intermedias las distribuciones son parecidas aunque ligeramente superiores en la muestra de hombres, lo que se explicaría por la tardía incorporación de la mujer al mercado de trabajo en España, de forma que las cohortes de mujeres mayores estaría claramente subrrepresentadas en el mercado laboral . En general, la muestra de mujeres es más joven (la media es de 37,6 años) que la de los hombres $(39,9)$, y mientras las mujeres que trabajan a tiempo parcial son algo más jóvenes que el conjunto de mujeres $(36,9)$, la media de los hombre que trabajan a tiempo parcial aumenta $(41,6)$ con respecto al conjunto de hombres. En todos los casos la proporción de inmigrantes está entre el 23 y el 25\% con la excepción del grupo de mujeres trabajando a tiempo parcial con un contrato temporal en donde la proporción de inmigrantes se dispara al $42.5 \%$.

TABLA 4.-Comparación conclusiones literatura y MCVL

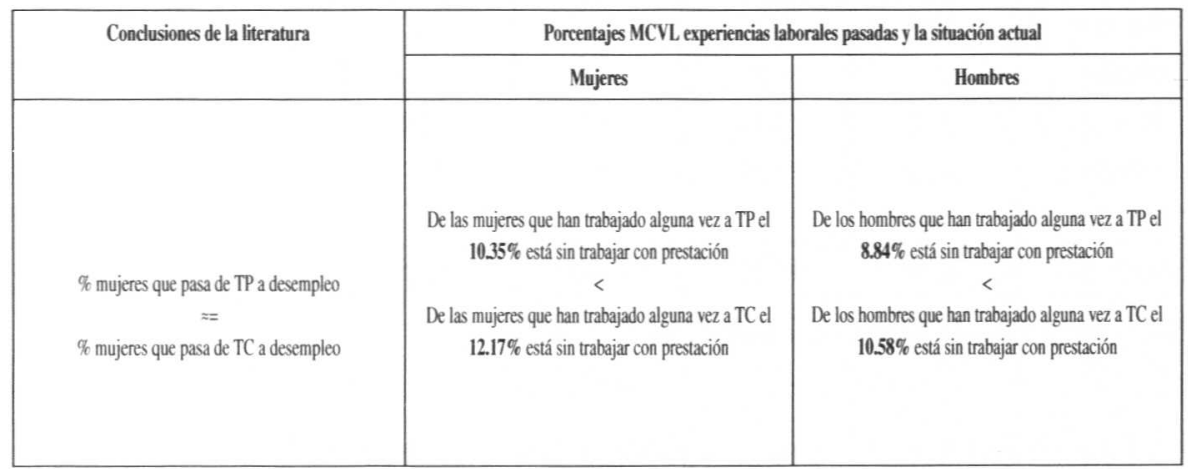


TABLA 4 (cont.).-Comparación conclusiones literatura y MCVL

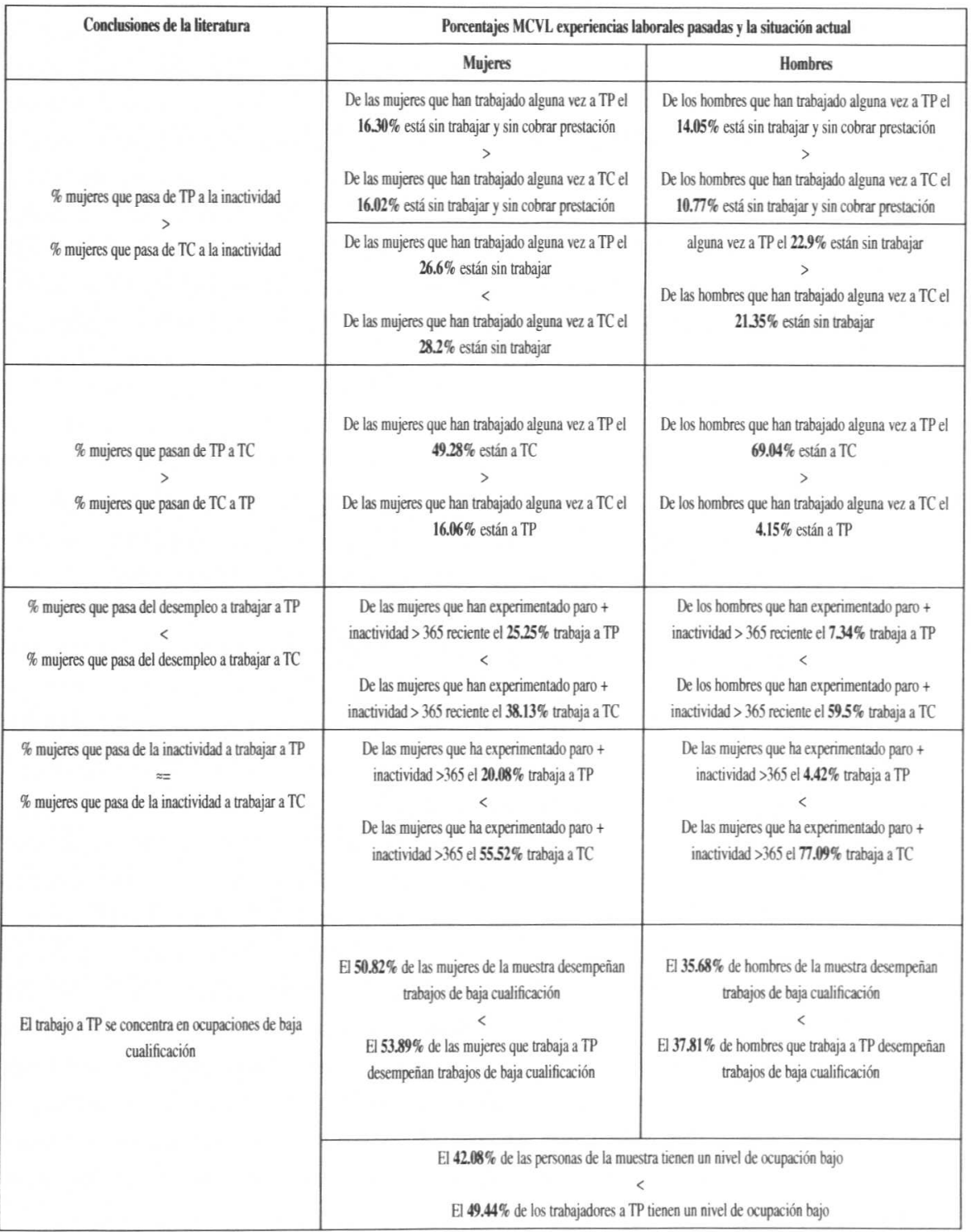

Fuentes: Buddelmeyer, Mourre y Ward-Warmedinger (2005), CES (1996) y Smith et al. (2000) y elaboración propia a partir de la MCVL 2007. 
Tomando como punto de partida la literatura sobre transiciones laborales en el mercado laboral español que repasamos en el apartado uno, la tabla 3 compara algunas de las conclusiones que obtiene dicha literatura (primera columna), basadas en el manejo de otras fuentes (fundamentalmente EPA y PHOGUE) con las que se pueden obtener del análisis de la muestra obtenida a partir de la MCVL (columnas segunda y tercera). Hay que tener en cuenta que se están comparado transiciones que, aunque muy parecidas, no son exactamente iguales pues la literatura sobre transiciones laborales tienen en cuenta experiencias laborales consecutivas mientras que las definiciones de experiencias laborales pasadas utilizadas aquí tienen en cuenta cualquier experiencia pasada como determinante de la situación actual. En todo caso, en términos generales, las conclusiones que se obtienen a partir de la MCVL son coherentes con las obtenidas por la literatura sobre el tema.

\section{LOS DETERMINANTES DE LAS PROBABILIDADES DE TERMINAR EL PERIODO DE MUESTREO TRABAJANDO A TIEMPO PARCIAL EN COMPARACIÓN A TERMINAR EN OTRA SITUACIÓN LABORAL}

\subsection{Especificación del modelo y variables explicativas de la situación laboral actual}

Con el objetivo de identificar los determinantes de terminar el periodo de muestreo trabajando a tiempo parcial, ya sea con un contrato indefinido o con un contrato temporal y compararlos con los determinantes del resto de situaciones laborales, hemos estimado seis modelos binomiales, uno para cada situación laboral. En todos ellos la variable dependiente es dicotómica y toma valor uno cuando el individuo finaliza el periodo de muestreo en una determinada situación laboral y cero en el resto de los casos. Las estimaciones asumen una función de distribución logística de la variable dependiente (finalizar o no en una determinada situación laboral) sobre las variables explicativas y presentan las estimaciones como efectos marginales. Es decir, para calcular el efectos de las características personales en las probabilidades de encontrase en una u otra situación, construimos seis variables dependientes, $P i$, binarias y con valor 1 ó 0 según el individuo finalice el muestreo en una situación o no. Posteriormente calculamos la regresión

$$
P_{i}=\left(X_{i} \times N\right) \quad \neg \mathrm{i}=1,2, \ldots, \mathrm{N}
$$




\section{Donde:}

$\varphi()=$. función de densidad.

$i=$ denota al individuo.

$X=$ vector $(1 \times \mathrm{Q})$ de características observables de cada individuo.

$\beta=$ vector $(Q \times 1)$ de coeficientes de cada característica individual.

Hemos preferido la estimación de cinco modelos binomiales, frente a la opción de estimar un modelo multinomial, porque la segunda opción precisaría de una jerarquización clara de la variable dependiente, en este caso, las seis situaciones laborales en las que han quedado encuadrados todos los individuos. Esto implicaría asumir que hay situaciones laborales preferibles/mejores a otras. No sería excesivo asumir que trabajar con un contrato indefinido es preferible a hacerlo con un contrato temporal y que no trabajar cobrando prestación por desempleo es preferible a no trabajar sin cobrarla. Sin embargo, aunque todo parece indicar que gran parte de los trabajadores a tiempo parcial en España lo hacen por falta de una opción a tiempo completo, nos parece excesivo asumir a priori que el trabajo a tiempo completo es preferible al trabajo a tiempo parcial y que no existe ningún tipo de voluntariedad entre los trabajadores a tiempo parcial.

Las tablas 4, 5 y 6 muestran los efectos marginales de la estimación del efecto que tienen las variables explicativas $(X s)$ sobre la probabilidad de que el trabajador se encuentre en cada una de las situaciones laborales descritas anteriormente. Como variables explicativas estamos utilizando las mismas que en el análisis de frecuencias del apartado anterior.

Las estimaciones se han calculado tanto para el conjunto de la muestra como para hombres y mujeres por separado ${ }^{8}$. En las regresiones que hemos realizado para el conjunto de la muestra observamos que las probabilidades de trabajar a tiempo completo son menores para las mujeres sobre todo si nos referimos a trabajar con un contrato indefinido. Por el contrario, ser mujer incrementa las probabilidades de terminar el muestreo trabajando a tiempo parcial o sin trabajar.

${ }^{8}$ El grupo de control es hombre que trabaja en los servicios, con un nivel de ocupación alto, que ha firmado entre 3 y 5 contratos, tiene entre 30 y 35 años, nacido en España, viviendo en una región con un PIB inferior a la media en una población menor de 40000 habitantes y en la misma provincia en la que nació. 
TABLA 5.-Determinantes de la probabilidad de trabajar a tiempo parcial

\begin{tabular}{|c|c|c|c|c|c|c|}
\hline & \multicolumn{3}{|c|}{ TP con contrato indefinido } & \multicolumn{3}{|c|}{ TP con contrato temporal } \\
\hline & H y M & Mujeres & Hombres & H у M & Mujeres & Hombres \\
\hline Género & $2.697^{*}$ & - & - & $1.833^{*}$ & - & - \\
\hline $\begin{array}{l}\text { Sector } \\
\text { agricultura } \\
\text { industria } \\
\text { construcción } \\
\text { comercio }\end{array}$ & $\begin{array}{l}0.209^{*} \\
0.475^{*} \\
0.374^{*} \\
1.426^{*}\end{array}$ & $\begin{array}{l}0.176^{*} \\
0.589^{*} \\
0.825^{*} \\
1.456^{*}\end{array}$ & $\begin{array}{l}0.202^{*} \\
0.328^{*} \\
0.182^{*} \\
1.091^{*}\end{array}$ & $\begin{array}{l}0.086^{*} \\
0.419^{*} \\
0.229^{*} \\
0.447^{*}\end{array}$ & $\begin{array}{l}0.076^{*} \\
0.152^{*} \\
0.363^{*} \\
0.441^{*}\end{array}$ & $\begin{array}{l}0.133^{*} \\
0.749^{*} \\
0.277^{*} \\
0.483^{*}\end{array}$ \\
\hline $\begin{array}{l}\text { Ocupación } \\
\text { Medio } \\
\text { Bajo }\end{array}$ & $\begin{array}{l}1.575^{*} \\
1.949^{*}\end{array}$ & $\begin{array}{l}1.857^{*} \\
2.16^{*}\end{array}$ & $\begin{array}{c}0.961 \\
1.252^{*}\end{array}$ & $\begin{array}{c}0.703^{*} \\
1.035^{* *}\end{array}$ & $\begin{array}{l}0.835^{*} \\
1.257^{*}\end{array}$ & $\begin{array}{l}0.67^{*} \\
1.012\end{array}$ \\
\hline Cambio S. & $0.879^{*}$ & $1.052^{*}$ & $0.605^{*}$ & $1.429^{*}$ & $1.366^{*}$ & $1.131^{*}$ \\
\hline $\begin{array}{l}\text { Contratos } \\
1 \\
2 \\
6-10 \\
11-20 \\
21-30 \\
\text { Más de } 30\end{array}$ & $\begin{array}{l}0.778^{*} \\
1.299^{*} \\
0.895^{*} \\
0.882^{*} \\
0.604^{*} \\
0.656^{*}\end{array}$ & $\begin{array}{c}0.861^{*} \\
0.943^{* *} \\
1.038^{* *} \\
0.946^{*} \\
0.65^{*} \\
0.658^{*}\end{array}$ & $\begin{array}{l}0.698^{*} \\
2.114^{*} \\
0.626^{*} \\
0.917^{*} \\
0.716^{*} \\
0.734^{*}\end{array}$ & $\begin{array}{l}0.661^{*} \\
0.587^{*} \\
1.132^{*} \\
1.229^{*} \\
1.105^{*} \\
1.039\end{array}$ & $\begin{array}{c}0.481^{*} \\
0.548^{*} \\
1.324^{*} \\
1.515^{*} \\
1.32^{*} \\
1.301^{*}\end{array}$ & $\begin{array}{c}1.024 \\
0.646^{*} \\
0.874^{*} \\
0.81^{*} \\
0.916^{* *} \\
0.747^{*}\end{array}$ \\
\hline $\begin{array}{l}>365 \\
\text { Últimos 5a. } \\
\text { Anterior }\end{array}$ & $\begin{array}{l}0.709^{*} \\
1.182^{*}\end{array}$ & $\begin{array}{l}0.664^{*} \\
1.118^{*}\end{array}$ & $\begin{array}{l}0.819^{*} \\
1.371^{*} \\
\end{array}$ & $\begin{array}{l}1.263^{*} \\
0.576^{*}\end{array}$ & $\begin{array}{l}1.331^{*} \\
0.641^{*}\end{array}$ & $\begin{array}{l}1.355^{*} \\
0.511^{*}\end{array}$ \\
\hline $\begin{array}{l}\text { Contratos } \\
\text { fijos_tp } \\
\text { temp_tc } \\
\text { temp_tp } \\
\text { fijos_tc } \\
\text { autónomo }\end{array}$ & $\begin{array}{c}3.423^{*} \\
0.441^{*} \\
1.751^{*} \\
0.937^{*} \\
0.975 \\
\end{array}$ & $\begin{array}{l}2.637^{*} \\
0.491^{*} \\
1.865^{*} \\
0.971^{*} \\
0.778^{*}\end{array}$ & $\begin{array}{l}6.31^{*} \\
0.387^{*} \\
1.312^{*} \\
0.787^{*} \\
1.771^{*}\end{array}$ & $\begin{array}{l}0.704^{*} \\
0.599^{*} \\
4.174^{*} \\
0.565^{*} \\
1.375^{*}\end{array}$ & $\begin{array}{l}0.657^{*} \\
0.668^{*} \\
3.582^{*} \\
0.319^{*} \\
1.615^{*}\end{array}$ & $\begin{array}{c}0.932^{* *} \\
0.608^{*} \\
5.511^{*} \\
1.206^{*} \\
0.899^{*}\end{array}$ \\
\hline $\begin{array}{l}\text { Edad } \\
<20 \\
21-25 \\
26-30 \\
36-40 \\
41-45 \\
46-50 \\
51-55 \\
56-60 \\
61-65 \\
+ \text { de } 65\end{array}$ & $\begin{array}{c}0.848^{* *} \\
1.15^{*} \\
0.904^{*} \\
1.145^{*} \\
1.179^{*} \\
0.979 \\
0.818^{*} \\
0.873^{*} \\
0.737^{*} \\
1.287^{*}\end{array}$ & $\begin{array}{c}0.646^{*} \\
0.737^{*} \\
0.841^{*} \\
1.069^{*} \\
1.399^{*} \\
1.14^{*} \\
0.925^{*} \\
1.018 \\
0.852^{*} \\
0.728^{*}\end{array}$ & $\begin{array}{c}1.829^{*} \\
2.638^{*} \\
1.248^{*} \\
1.473^{*} \\
0.787^{*} \\
0.71^{*} \\
0.739^{*} \\
0.736^{*} \\
0.732^{*} \\
2.333^{*}\end{array}$ & $\begin{array}{c}1.825^{*} \\
0.962 \\
0.817^{*} \\
0.775^{*} \\
0.394^{*} \\
0.988 \\
0.935^{*} \\
0.629^{*} \\
8.26^{*} \\
0.859\end{array}$ & $\begin{array}{c}1.203^{* *} \\
0.806^{*} \\
0.842^{*} \\
0.498^{*} \\
0.296^{*} \\
0.641^{*} \\
0.845^{*} \\
0.53^{*} \\
2.293^{*} \\
0.637^{*}\end{array}$ & $\begin{array}{c}4.035^{*} \\
1.736^{*} \\
0.913^{* *} \\
1.931^{*} \\
0.707^{*} \\
2.647^{*} \\
0.86^{*} \\
1.09 \\
24.145^{*} \\
1.535^{*}\end{array}$ \\
\hline Emigrante & $0.819^{*}$ & $0.631^{*}$ & $1.923^{*}$ & $1.617^{*}$ & $2.21^{*}$ & $0.759^{*}$ \\
\hline
\end{tabular}


TABLA 5 (cont.).-Determinantes de la probabilidad de trabajar a tiempo parcial

\begin{tabular}{|l|c|c|c|c|c|c|}
\hline & \multicolumn{3}{|c|}{ TP con contrato indefinido } & \multicolumn{3}{c|}{ TP con contrato temporal } \\
\hline & H y M & Mujeres & Hombres & H y M & Mujeres & Hombres \\
\hline Contexto & & & & & & \\
PIB & $0.863^{*}$ & 1.017 & $0.554^{*}$ & $0.96^{*}$ & 0.99 & $0.863^{*}$ \\
$>40000$ & $0.885^{*}$ & $0.932^{*}$ & $0.783^{*}$ & $1.158^{*}$ & $1.177^{*}$ & $1.063^{*}$ \\
Provincia $\neq$ & $0.897^{*}$ & $0.897^{*}$ & $0.872^{*}$ & $1.208^{*}$ & $0.883^{*}$ & $1.728^{*}$ \\
\hline pseudos $R^{2}$ & 0.1469 & 0.0846 & 0.199 & 0.1801 & 0.1764 & 0.1991 \\
\hline Núm. Obs. & 559453 & 246347 & 313106 & 559453 & 246347 & 313106 \\
\hline
\end{tabular}

* Significativo al 95\%

** Significativo al $90 \%$

TABLA 6.-Determinantes de la probabilidad de trabajar a tiempo completo

\begin{tabular}{|c|c|c|c|c|c|c|}
\hline & \multicolumn{3}{|c|}{ TC con contrato indefinido } & \multicolumn{3}{|c|}{ TC con contrato temporal } \\
\hline & H y M & Mujeres & Hombres & Hy M & Mujeres & Hombres \\
\hline Género & $0.568^{*}$ & - & - & $0.723^{*}$ & - & - \\
\hline $\begin{array}{l}\text { Sector } \\
\text { agricultura } \\
\text { industria } \\
\text { construcción } \\
\text { comercio }\end{array}$ & $\begin{array}{l}0.512^{*} \\
2.013^{*} \\
0.398^{*} \\
1.819^{*}\end{array}$ & $\begin{array}{l}0.321^{*} \\
2.417^{*} \\
0.743^{*} \\
1.683^{*}\end{array}$ & $\begin{array}{l}0.592^{*} \\
1.803^{*} \\
0.391^{*} \\
2.051^{*}\end{array}$ & $\begin{array}{c}1.291^{*} \\
0.843^{*} \\
2.558^{*} \\
0.58^{*}\end{array}$ & $\begin{array}{c}1.051 \\
1.188^{*} \\
2.581^{*} \\
0.479^{*}\end{array}$ & $\begin{array}{l}1.359^{*} \\
0.726^{*} \\
2.435^{*} \\
0.671^{*}\end{array}$ \\
\hline $\begin{array}{l}\text { Ocupación } \\
\text { Medio } \\
\text { Bajo }\end{array}$ & $\begin{array}{l}0.805^{*} \\
0.632^{*}\end{array}$ & $\begin{array}{l}0.888^{*} \\
0.721^{*}\end{array}$ & $\begin{array}{l}0.785^{*} \\
0.577^{*}\end{array}$ & $\begin{array}{l}0.817^{*} \\
0.913^{*}\end{array}$ & $\begin{array}{l}0.509^{*} \\
0.633^{*}\end{array}$ & $\begin{array}{c}1.288^{*} \\
1.37^{*}\end{array}$ \\
\hline Cambio $S$. & $0.916^{*}$ & $0.922 *$ & $0.904^{*}$ & $1.103^{*}$ & $1.231^{*}$ & $1.03^{*}$ \\
\hline $\begin{array}{l}\text { Contratos } \\
1 \\
2 \\
6-10 \\
11-20 \\
21-30 \\
\text { Más de } 30\end{array}$ & $\begin{array}{c}1.203^{*} \\
1.497^{*} \\
0.88^{*} \\
0.609^{*} \\
0.462^{*} \\
0.279^{*}\end{array}$ & $\begin{array}{l}1.032 \\
1.413^{*} \\
0.839^{*} \\
0.593^{*} \\
0.578^{*} \\
0.391^{*}\end{array}$ & $\begin{array}{l}1.392^{*} \\
1.424^{*} \\
0.908^{*} \\
0.607^{*} \\
0.373^{*} \\
0.218^{*}\end{array}$ & $\begin{array}{l}0.403^{*} \\
0.618^{*} \\
1.165^{*} \\
1.627^{*} \\
1.867^{*} \\
2.159^{*}\end{array}$ & $\begin{array}{c}0.292^{*} \\
0.658^{*} \\
0.974 \\
1.6^{*} \\
1.832^{*} \\
2.399^{*}\end{array}$ & $\begin{array}{c}0.488^{*} \\
0.506^{*} \\
1.426^{*} \\
1.756^{*} \\
2.09^{*} \\
2.162^{*}\end{array}$ \\
\hline $\begin{array}{l}>365 \\
\text { Últimos 5a. } \\
\text { Anterior }\end{array}$ & $\begin{array}{l}0.368^{*} \\
1.079^{*} \\
\end{array}$ & $\begin{array}{l}0.319^{*} \\
1.076^{*}\end{array}$ & $\begin{array}{l}0.409^{*} \\
1.068^{*} \\
\end{array}$ & $\begin{array}{l}1.262^{*} \\
0.879^{*}\end{array}$ & $\begin{array}{l}1.265^{*} \\
0.918^{*}\end{array}$ & $\begin{array}{l}1.235^{*} \\
0.881^{*}\end{array}$ \\
\hline $\begin{array}{l}\text { Contratos } \\
\text { fijos_tp } \\
\text { temp_tc } \\
\text { temp_tp } \\
\text { fijos_tc } \\
\text { autónomo }\end{array}$ & $\begin{array}{l}0.598^{*} \\
0.666^{*} \\
0.683^{*} \\
1.231^{*} \\
0.894^{*}\end{array}$ & $\begin{array}{c}0.666^{*} \\
0.702^{*} \\
0.575^{*} \\
1.57^{*} \\
0.858^{*}\end{array}$ & $\begin{array}{c}0.504^{*} \\
0.643^{*} \\
0.79^{*} \\
0.986 \\
0.863^{*} \\
\end{array}$ & $\begin{array}{l}0.768^{*} \\
3.244^{*} \\
0.869^{*} \\
0.673^{*} \\
1.113^{*} \\
\end{array}$ & $\begin{array}{l}0.836^{*} \\
3.675^{*} \\
0.739^{*} \\
0.57^{*} \\
1.27^{*} \\
\end{array}$ & $\begin{array}{l}0.718^{*} \\
2.893^{*} \\
0.971^{*} \\
0.729^{*} \\
1.165^{*}\end{array}$ \\
\hline
\end{tabular}


TABLA 6 (cont.).-Determinantes de la probabilidad de trabajar a tiempo completo

\begin{tabular}{|l|c|c|c|c|c|c|}
\hline & \multicolumn{3}{|c|}{ TC con contrato indefinido } & \multicolumn{3}{c|}{ TC con contrato temporal } \\
\hline & H y M & Mujeres & Hombres & H y M & Mujeres & Hombres \\
\hline Edad & & & & & & \\
$<20$ & $0.109^{*}$ & $0.134^{*}$ & $0.093^{*}$ & $1.662^{*}$ & $1.723^{*}$ & $1.83^{*}$ \\
$21-25$ & $0.353^{*}$ & $0.369^{*}$ & $0.306^{*}$ & $1.285^{*}$ & $1.527^{*}$ & $1.198^{*}$ \\
$26-30$ & $0.742^{*}$ & $0.748^{*}$ & $0.694^{*}$ & $1.383^{*}$ & $1.422^{*}$ & $1.356^{*}$ \\
$36-40$ & $1.070^{*}$ & $1.357^{*}$ & $0.883^{*}$ & $0.902^{*}$ & $0.831^{*}$ & $0.973^{* *}$ \\
$41-45$ & 0.985 & $0.924^{*}$ & $1.118^{*}$ & $0.925^{*}$ & $0.825^{*}$ & $0.956^{*}$ \\
$46-50$ & $1.176^{*}$ & 0.974 & $1.508^{*}$ & $0.626^{*}$ & $0.935^{*}$ & $0.469^{*}$ \\
$51-55$ & $0.957^{*}$ & $0.855^{*}$ & $1.119^{*}$ & $0.798^{*}$ & $0.618^{*}$ & $0.903^{*}$ \\
$56-60$ & $0.843^{*}$ & $0.713^{*}$ & 0.982 & $0.532^{*}$ & $0.519^{*}$ & $0.551^{*}$ \\
$61-65$ & $0.279^{*}$ & $0.347^{*}$ & $0.258^{*}$ & $0.411^{*}$ & $0.45^{*}$ & $0.420^{*}$ \\
+ de 65 & $0.084^{*}$ & $0.136^{*}$ & $0.068^{*}$ & $0.191^{*}$ & $0.204^{*}$ & $0.194^{*}$ \\
\hline Emigrante & $0.417^{*}$ & $0.597^{*}$ & $0.27^{*}$ & $1.623^{*}$ & $1.058^{*}$ & $2.353^{*}$ \\
\hline Contexto & & & & & & \\
PIB & $1.49^{*}$ & $1.289^{*}$ & $1.664^{*}$ & $0.772^{*}$ & $0.68^{*}$ & $0.817^{*}$ \\
$>40$ 000 & $1.213^{*}$ & $1.19^{*}$ & $1.186^{*}$ & $1.045^{*}$ & $1.103^{*}$ & 1.008 \\
Provincia $\neq$ & $0.947^{*}$ & $1.07^{*}$ & $0.87^{*}$ & $0.931^{*}$ & $0.855^{*}$ & 0.991 \\
\hline pseudos $R^{2}$ & 0.1991 & 0.1724 & 0.2245 & 0.1415 & 0.1244 & 0.1611 \\
\hline Núm. Obs. & 559453 & 246347 & 313106 & 559453 & 246347 & 313106 \\
\hline
\end{tabular}

* Significativo al $95 \%$

** Significativo al $90 \%$

TABLA 7.-Determinantes de la probabilidad de terminar el período de muestreo sin trabajar

\begin{tabular}{|l|c|c|c|c|c|c|}
\hline & \multicolumn{3}{|c|}{ Sin trabajar y cobrando prestación por } & \multicolumn{3}{c|}{ Sin trabajar y sin cobrar prestación por } \\
desempleo & \multicolumn{3}{|c|}{ desempleo } \\
\hline & H y M & Mujeres & Hombres & H y M & Mujeres & Hombres \\
\hline Género & $1.903^{*}$ & - & - & 1.308 & - & - \\
\hline Sector & & & & & & \\
agricultura & $3.231^{*}$ & $4.899^{*}$ & $2.226^{*}$ & $0.940^{*}$ & $0.901^{*}$ & 0.973 \\
industria & 0.985 & 0.985 & $0.95^{*}$ & $0.46^{*}$ & $0.414^{*}$ & $0.5^{*}$ \\
construcción & $1.372^{*}$ & 0.986 & $1.298^{*}$ & $1.382^{*}$ & $0.675^{*}$ & $1.34^{*}$ \\
comercio & 0.979 & $1.070^{*}$ & $0.771^{*}$ & $0.599^{*}$ & $0.806^{*}$ & $0.39^{*}$ \\
\hline Ocupación & & & & & & \\
Medio & $2.683^{*}$ & $4.922^{*}$ & $1.410^{*}$ & $1.077^{*}$ & $0.771^{*}$ & $1.529^{*}$ \\
Bajo & $2.703^{*}$ & $3.945^{*}$ & $1.897^{*}$ & $1.125^{*}$ & $0.838^{*}$ & $1.642^{*}$ \\
\hline Cambio S. & $1.134^{*}$ & $0.952^{*}$ & $1.666^{*}$ & $0.907^{*}$ & $0.677^{*}$ & $1.23^{*}$ \\
\hline
\end{tabular}


DETERMINANTES DE LA SITUACIÓN LABORAL EN ESPAÑA:

TABLA 7 (cont.).-Determinantes de la probabilidad de terminar el período de muestreo sin trabajar

\begin{tabular}{|c|c|c|c|c|c|c|}
\hline & \multicolumn{3}{|c|}{$\begin{array}{c}\begin{array}{c}\text { Sin trabajar y cobrando prestación por } \\
\text { desempleo }\end{array} \\
\end{array}$} & \multicolumn{3}{|c|}{$\begin{array}{l}\text { Sin trabajar y sin cobrar prestación por } \\
\text { desempleo }\end{array}$} \\
\hline & H y M & Mujeres & Hombres & $\mathrm{H}$ y M & Mujeres & Hombres \\
\hline $\begin{array}{l}\text { Contratos } \\
1 \\
2 \\
6-10 \\
11-20 \\
21-30 \\
\text { Más de } 30\end{array}$ & $\begin{array}{c}1.784^{*} \\
1.593^{*} \\
0.872^{*} \\
1.05^{*} \\
1.474 * \\
1.78^{*}\end{array}$ & $\begin{array}{c}1.533^{*} \\
1.843^{*} \\
0.817^{*} \\
0.993 \\
1.24^{*} \\
0.917^{*}\end{array}$ & $\begin{array}{c}2.262^{*} \\
1.24^{*} \\
0.909^{*} \\
1.122^{*} \\
1.702^{*} \\
2.707^{*}\end{array}$ & $\begin{array}{c}1.864^{*} \\
0.603^{*} \\
1.07^{*} \\
1.097^{*} \\
1.329^{*} \\
1.909^{*}\end{array}$ & $\begin{array}{c}2.912^{*} \\
0.564^{*} \\
1.205^{*} \\
1.038^{* *} \\
1.143^{*} \\
1.717^{*}\end{array}$ & $\begin{array}{l}1.021 \\
0.736^{*} \\
0.888^{*} \\
1.111^{*} \\
1.476^{*} \\
2.02^{*}\end{array}$ \\
\hline $\begin{array}{l}>365 \\
\text { Últimos 5a. } \\
\text { Anterior }\end{array}$ & $\begin{array}{c}2.10^{*} \\
1.453^{*}\end{array}$ & $\begin{array}{c}2.05^{*} \\
1.351^{*}\end{array}$ & $\begin{array}{l}2.147^{*} \\
1.579^{*}\end{array}$ & $\begin{array}{l}2.397 * \\
0.88^{*}\end{array}$ & $\begin{array}{l}2.835^{*} \\
0.912^{*}\end{array}$ & $\begin{array}{l}2.075^{*} \\
0.857^{*}\end{array}$ \\
\hline $\begin{array}{l}\text { Contratos } \\
\text { fijos_tp } \\
\text { temp_tc } \\
\text { temp_tp } \\
\text { fijos_tc } \\
\text { autónomo }\end{array}$ & $\begin{array}{c}1.676^{*} \\
2.18^{*} \\
0.989 \\
1.343^{*} \\
0.921^{*}\end{array}$ & $\begin{array}{c}1.97 * \\
2.459 * \\
1.042 * \\
1.277 * \\
0.971\end{array}$ & $\begin{array}{c}0.969 \\
1.697^{*} \\
0.967^{*} \\
1.462^{*} \\
0.912^{*}\end{array}$ & $\begin{array}{c}0.995 \\
1.191^{*} \\
1.313^{*} \\
1.4^{*} \\
1.109^{*}\end{array}$ & $\begin{array}{l}0.621^{*} \\
1.343^{*} \\
1.606^{*} \\
1.363^{*} \\
1.008\end{array}$ & $\begin{array}{l}2.206^{*} \\
1.124^{*} \\
1.185^{*} \\
1.509^{*} \\
1.15^{*}\end{array}$ \\
\hline $\begin{array}{l}\text { Edad } \\
<20 \\
21-25 \\
26-30 \\
36-40 \\
41-45 \\
46-50 \\
51-55 \\
56-60 \\
61-65 \\
+ \text { de } 65\end{array}$ & $\begin{array}{l}0.81^{*} \\
1.317^{*} \\
1.418^{*} \\
1.202^{*} \\
0.917^{*} \\
1.327^{*} \\
1.872^{*} \\
3.503^{*} \\
5.448^{*} \\
2.139^{*}\end{array}$ & $\begin{array}{l}0.687^{*} \\
1.367^{*} \\
1.647^{*} \\
1.407^{*} \\
0.896^{*} \\
1.263^{*} \\
2.432^{*} \\
3.976^{*} \\
5.208^{*} \\
1.36^{*} \\
\end{array}$ & $\begin{array}{c}0.876 \\
1.275^{*} \\
1.115^{*} \\
0.922^{*} \\
0.868^{*} \\
1.139^{*} \\
1.244^{*} \\
2.702^{*} \\
4.710^{*} \\
2.331^{*} \\
\end{array}$ & $\begin{array}{c}8.786^{*} \\
3.44^{*} \\
1.159^{*} \\
0.965^{*} \\
1.585^{*} \\
1.033 \\
0.835^{*} \\
0.92^{*} \\
1.314^{*} \\
22.317^{*}\end{array}$ & $\begin{array}{c}9.059^{*} \\
3.252^{*} \\
1.067^{*} \\
0.827^{*} \\
2.228^{*} \\
1.25^{*} \\
0.916^{*} \\
1.013 \\
1.344^{*} \\
18.756^{*} \\
\end{array}$ & $\begin{array}{c}9.203^{*} \\
3.666^{*} \\
1.295^{*} \\
1.051^{*} \\
1.063^{*} \\
0.913^{*} \\
0.767^{*} \\
0.85^{*} \\
1.281^{*} \\
25.999^{*} \\
\end{array}$ \\
\hline Emigrante & $1.225^{*}$ & $0.858^{*}$ & $1.886^{*}$ & $2.039 *$ & $2.192^{*}$ & $1.999 *$ \\
\hline $\begin{array}{l}\text { Contexto } \\
\text { PIB } \\
>40000 \\
\text { Provincia } \neq\end{array}$ & $\begin{array}{c}0.78^{*} \\
0.786^{*} \\
1.339^{*}\end{array}$ & $\begin{array}{l}0.921^{*} \\
0.816^{*} \\
1.513^{*}\end{array}$ & $\begin{array}{c}0.658^{*} \\
0.76^{*} \\
1.122^{*}\end{array}$ & $\begin{array}{l}0.878^{*} \\
0.807^{*} \\
0.948^{*}\end{array}$ & $\begin{array}{l}0.907^{*} \\
0.735^{*} \\
0.871^{*}\end{array}$ & $\begin{array}{c}0.844^{*} \\
0.924^{*} \\
1.029\end{array}$ \\
\hline pseudos $R^{2}$ & 0.1127 & 0.1169 & 0.1192 & 0.1038 & 0.1176 & 0.1219 \\
\hline Núm. Obs. & 559453 & 246347 & 313106 & 559453 & 246347 & 313106 \\
\hline
\end{tabular}

* Significativo al $95 \%$

** Significativo al $90 \%$

Las probabilidades de terminar el periodo de muestreo en una situación u otra varían según el sector en el que se trabaje. Los hombres y mujeres que más probabilidades 
tienen de trabajar a tiempo parcial con un contrato indefinido son los del sector comercial y los que tienen más probabilidades de trabajar a tiempo parcial con un contrato temporal son los del sector servicios. En lo que se refiere al tiempo completo, el sector con más probabilidades de trabajo a tiempo completo indefinido para los hombres es el industrial y para las mujeres el comercio y el que más probabilidades tiene de trabajo a tiempo completo temporal es, tanto para hombres como para mujeres, la construcción. Las mayores probabilidades de terminar sin trabajar y cobrando prestación por desempleo se encuentran, para ambos sexos y con mucha diferencia, en la agricultura. Finalmente, el sector con más probabilidades de terminar sin trabajar y sin cobrar prestación es el sector servicios para las mujeres y el de la construcción para los hombres.

El desempeñar una ocupación con un nivel de cualificación bajo aumenta las probabilidades de trabajar a tiempo parcial tanto con carácter indefinido como temporal y tanto para hombres como para mujeres. Este efecto es mayor para las mujeres que para los hombres y en el caso de los contratos indefinidos. Este resultado confirma que el trabajo a tiempo parcial en el mercado laboral español predominan los trabajadores más vulnerables y con menos opciones: mujeres y trabajadores con niveles de cualificación bajos. Sin embargo, el tener un nivel de cualificación bajo, no es significativo con respecto a tener un nivel alto cuando se estiman los determinantes masculinos del trabajo temporal a TP. Comparando estos resultados con los obtenidos para el trabajo a tiempo completo, observamos dos resultados relevantes. Primero que a mayor nivel de cualificación ocupacional, mayores probabilidades de trabajar a tiempo completo con un contrato indefinido tanto para hombres como para mujeres. Y segundo, que el nivel de cualificación afecta de distinta manera a las probabilidades que hombres y mujeres tienen de trabajar a tiempo completo con un contrato temporal. Mientras para los hombres tener niveles de cualificación medios y bajos aumenta las probabilidades de ser temporales a tiempo completo con respecto a tener el nivel alto, para las mujeres tener niveles de cualificación medios y bajos disminuye las probabilidades de ser temporales a tiempo completo con respecto a tener un nivel alto. En casi todos los casos, las probabilidades de terminar el periodo de muestreo sin trabajar aumentan a medida que disminuye el nivel de cualificación, sobre todo en el caso de las mujeres y especialmente en el caso de terminar cobrando la prestación por desempleo. La excepción la encontramos también en el caso de las mujeres pues sus probabilidades de encontrarse sin trabajar y sin cobrar prestación son mayores si su último puesto de trabajo implicaba un nivel de cualificación alto.

En general, las personas que han cambiado alguna vez de sector de actividad tienen menores probabilidades de trabajar con un contrato indefinido, tanto a tiempo parcial como a tiempo completo. La excepción a esta regla la encontramos en la probabilidad 
femenina de trabajar a tiempo parcial con un contrato indefinido que aumenta ligeramente si se ha cambiado de sector. El efecto de haber cambiado de sector en terminar el periodo de muestreo sin trabajar es distinto entre los hombres y las mujeres. Entre los primeros, cambiar de sector aumenta dichas probabilidades, entre las segundas las disminuye.

El efecto del número de contratos que se ha firmado a lo largo de la vida laboral en las probabilidades de trabajar a tiempo parcial difiere claramente entre hombres y mujeres. Comenzando con el trabajo a tiempo parcial indefinido, las probabilidades femeninas de trabajar a tiempo parcial con un contrato indefinido van aumentando paulatinamente a medida que se firman más contratos. Esto ocurre hasta el contrato 10, a partir del contrato 11 dichas probabilidades disminuyen. En el caso de los hombres las probabilidades solo son positivas para los que han firmado 2 contratos pero el descenso de las probabilidades a medida que se firman más contratos no es continuo: el impacto negativo de firmar entre 6 y 10 contratos, con respecto a firmar entre 3 y 5 , es mucho más fuerte que el de firmar entre 11 y 20 y muy parecido a los de los intervalos establecidos por encima de los 21 contratos. Por tanto, los contratos indefinidos a tiempo parcial son algo propio de mujeres que han firmado un número intermedio de contratos y de hombres que han firmado como mucho dos contratos. Mientras las probabilidades femeninas descienden de manera clara a partir de un determinado número de contratos (11), la evolución de las probabilidades de trabajar de manera indefinida a tiempo parcial a medida que aumenta el número de contratos es muy poco esclarecedora entre los hombres. En lo que se refiere al trabajo temporal a tiempo parcial, las probabilidades femeninas de trabajar a tiempo parcial con un contrato temporal se concentran entre el décimo primer contrato y el vigésimo y en los hombres entre el tercero y el quinto. La relación número de contratos-situación laboral es mucho más clara en las situaciones a tiempo completo. Tanto para hombres como para mujeres las probabilidades de conseguir un contrato indefinido a tiempo completo aumenta hasta el segundo contrato y disminuyen de manera continua a partir del tercero. Por el contrario, también tanto para hombres como para mujeres, las probabilidades de terminar el periodo muestreo con un contrato temporal a tiempo completo aumentan a medida que aumenta el número de contratos que han firmado. Estos resultados son una prueba evidente del atrapamiento en la temporalidad, mediante sucesivos contratos, en el trabajo a tiempo completo, y de que es un fenómeno que afecta tanto a hombres como a mujeres.

Las experiencias de desempleo e inactividad de más de un año de duración que han tenido lugar en los últimos cinco años aumentan las probabilidades de ocupar un puesto de trabajo temporal, tanto a tiempo completo como a tiempo parcial y tanto para los hombres como para las mujeres. Del mismo modo, para ambos sexos dichas 
experiencias disminuyen las probabilidades de ocupar un puesto de trabajo indefinido sobre todo si este es a tiempo completo. Por el contrario, las personas que han experimentado este tipo de situaciones hace más de cinco años tienen, ligeramente, más probabilidades de ser indefinidos. Interpretando estos resultados en términos de transiciones laborales, podemos decir que la transición del paro y/o la inactividad que ha tenido lugar en los últimos cinco años, al trabajo indefinido, sobre todo si este es a tiempo completo, es menos probable que la transición al trabajo temporal. Sin embargo, el efecto negativo de estas experiencias se difumina cuando se alejan en el tiempo, es decir, cuando han tenido lugar hace más de cinco años. Finalmente, es importante señalar que las experiencias de paro e inactividad de los últimos cinco años multiplican por dos las probabilidades de terminar el periodo de muestreo sin trabajar y por tres las probabilidades de las mujeres de terminar sin trabajar y sin cobrar prestación.

Continuando con el análisis de las transiciones laborales, los resultados resaltan la especial importancia de las variables explicativas que identifican relaciones laborales anteriores. A diferencia de cómo se plantean otros estudios, en el que nos ocupa al definir estas variables no sólo se tiene en cuenta la relación inmediatamente anterior a la situación actual sino todas las experiencias laborales pasadas. Por tanto, miden el efecto que en la situación actual tiene haber trabajado, con un contrato fijo a tiempo parcial (fijo_tp), con un contrato fijo a tiempo completo (fijo_tc), con un contrato temporal a tiempo parcial (temp_tp) y/o con un contrato temporal a tiempo completo (temp_tc).

Comenzando con los determinantes del trabajo a tiempo parcial indefinido, observamos que, tanto para hombres como para mujeres, el haber trabajado con anterioridad a tiempo parcial, tanto con carácter temporal como indefinido, tienen un efecto positivo. En este sentido, encontramos una diferencia importante con respecto a los determinantes del trabajo a tiempo completo indefinido en lo que se refiere al atrapamiento en la temporalidad. Mientras haber trabajado con un contrato temporal a tiempo parcial aumenta las probabilidades de trabajar a tiempo parcial indefinido, haber trabajado con un contrato temporal a tiempo completo reduce las probabilidades de trabajar a tiempo completo indefinido. Por último, haber sido autónomo tiene un efecto positivo en trabajar indefinido a tiempo parcial entre los hombres pero no entre las mujeres. Detrás de este resultado pueden esconderse vidas laborales en las que se compagina el ser autónomo con ser trabajador a tiempo parcial. Por último, entre las mujeres haber tenido un contrato indefinido a tiempo completo tienen un efecto cercano a cero, apenas disminuye las probabilidades de trabajar de forma indefinida a tiempo parcial en un 3\% mientras el impacto para los hombres está cercano al 22\%. Esto muestra que las transiciones de TC indefinido a TP indefinido son mucho más comunes entre las mujeres. Seguramente, detrás de este resultado, se esconden circunstancias de la vida 
familiar, su conciliación con la vida profesional y la decisión de interrumpir una carrera profesional en la que ya se había alcanzado un contrato indefinido a TC.

En lo que se refiere a los determinantes del trabajo a tiempo parcial temporal, volvemos a encontrar que, tanto entre hombres como entre mujeres, los que tienen mayores probabilidades de finalizar el periodo de muestreo con un contrato de este tipo son los que ya lo han tenido con anterioridad. A diferencia del caso anterior, ahora, entre las mujeres tienen un efecto positivo haber sido autónomas. De ser cierta la interpretación anterior, cuando las mujeres compaginan trabajo autónomo con contratos a tiempo parcial lo harían con contratos temporales pero cuando los hombres compaginan trabajo autónomo con contratos a tiempo parcial lo hacen con contratos indefinidos. Además, también se cambian las tornas en cuanto al efecto de haber tenido un contrato indefinido a tiempo completo que ahora tiene un efecto positivo para los hombres y muy negativo para las mujeres. Como veremos al estudiar los coeficientes de los intervalos de edad, lo que está detrás de este resultado es que para muchos hombres los contratos temporales a tiempo parcial son la antesala de la jubilación (véase tabla 3). En comparación con los determinantes del trabajo temporal a tiempo completo, observamos que sólo haber sido con anterioridad trabajador temporal a tiempo completo tiene un efecto positivo en las probabilidades de serlo a cierre de muestra.

Por último, los trabajadores que más probabilidades tienen de terminar sin trabajar cobrando prestación por desempleo son, en este orden, los que, antes de dejar de trabajar, han sido alguna vez trabajadores temporales a tiempo completo, los fijos a tiempo completo y, entre las mujeres, las que son fijas a tiempo parcial. En cuanto a terminar sin trabajar y sin cobrar prestación, las mayores probabilidades se dan entre las mujeres que han sido alguna vez trabajadoras temporales a tiempo parcial y entre los hombres que han sido alguna vez trabajadores fijos a tiempo parcial.

La edad afecta de manera muy distinta a las probabilidades de que hombres y mujeres trabajen a tiempo parcial. A medida que aumenta la edad aumentan las probabilidades de que las mujeres trabajen a tiempo parcial con un contrato indefinido, sin embargo, esta tendencia cambia a partir del intervalo de edad comprendido entre los 41 y 45 años en el que las probabilidades de que una mujer se encuentren en esta situación laboral alcanzan su máximo y comienzan a disminuir. En el caso de los hombres las mayores probabilidades se concentran tanto en edades muy bajas, menores de 25 , como en edades muy altas, mayores de 65. Sin embargo, los resultados de los determinantes del trabajo temporal tiempo parcial muestran perfiles más parecidos para hombres y mujeres: mayores probabilidades a edades bajas y altas. Adicionalmente se observa un efecto positivo muy fuerte entre los hombres que rompe esta tendencia: los hombres que tienen entre 46 y 50 años tienen mayores probabilidades de trabajar 
a tiempo parcial con contrato temporal que los inmediatamente más jóvenes, entre 30 y 45 , y los inmediatamente más mayores, entre 51 y 60 . Los resultados permiten concluir que el trabajo a tiempo parcial tiene tres perfiles diferenciados en cuanto a su distribución por edades.

En primer lugar, jóvenes que seguramente compaginan el trabajo con los estudios, aunque esto es algo que no podemos contrastar con la MCVL, y que, en el caso de los hombres trabajan a tiempo parcial tanto con contrato temporal como indefinido $\mathrm{y}$, en el de las mujeres, mayoritariamente de forma temporal. Este perfil de trabajo temporal es el que ha llevado a pensar que el trabajo a TP puede ser una vía de entrada en el mercado de trabajo, sin embargo, en las estimaciones que hemos realizado, no se observa que haber trabajado con anterioridad a TP aumente las probabilidades de trabajar hoy a tiempo completo. Quizá un estudio específico para jóvenes pudiera completar la información y las interpretaciones que hemos obtenido.

En segundo lugar, personas que a edades intermedias trabajan a tiempo parcial. Se tiende a dar por hecho que este perfil es acaparado en su mayoría por mujeres. Los resultados corroboran que, en gran medida, así es y que estas mujeres tienden a encontrar cierta estabilidad en el trabajo tiempo parcial pues este fenómeno se observa fundamentalmente en el trabajo a TP que tiene carácter indefinido. Además se encuentra evidencias de que este tipo de mujeres «renuncia», en muchos casos, a una estabilidad que ya tenía a tiempo completo: recordemos que haber trabajado con un contrato fijo a tiempo completo sólo reduce las probabilidades femeninas de trabajar fija a TP en un $3 \%$ mientras que en el caso de los hombres las reduce en un $22 \%$. Sin embargo, aunque el trabajo indefinido a tiempo parcial entre los hombres es algo claramente propio de personas muy jóvenes y muy mayores, el trabajo temporal a TP también lo es de hombres que tienen entre 46 y 50 años. Puede ocurrir que, en determinados contextos familiares, sea el hombre el que decida trabajar temporalmente a tiempo parcial para asumir las cargas familiares. La MCVL no ofrece información suficiente sobre la vida familiar como para contrastar esta hipótesis.

En tercer lugar, personas que trabajan a tiempo parcial al final de su carrera como antesala de la jubilación. Este fenómeno es básicamente masculino y en general bajo contratos fijos aunque también se observa, de manera mucho más moderada, entre las mujeres. Este fenómeno es propio de personas con alto nivel ocupacional (casi el $80 \%$ de los hombres mayores de 61 años que trabajan con un contrato fijo a TP, tienen un nivel de cualificación alto) lo que explica que el tener un nivel de cualificación bajo no sea significativo con respecto a tener un nivel de ocupación alto cuando se estiman los determinantes masculinos del trabajo temporal a TP. 
Ser inmigrante afecta de manera totalmente distinta a hombres y mujeres. Mientras multiplica por dos las probabilidades de que un hombre trabaje con contrato indefinido a TP, reduce en casi un $40 \%$ las probabilidades de que lo haga una mujer. Mientras multiplica por más de dos las probabilidades de que una mujer trabaje temporal a TP, reduce en casi un $25 \%$ las probabilidades de que lo haga un hombre. Los hombres inmigrantes tienden más a trabajar fijos a tiempo parcial y menos con contrato temporal a TP que los nacionales. Las mujeres inmigrantes tienden a trabajar más temporales a tiempo parcial y menos con contrato indefinido a TP que las nacionales. En cuanto al resto de situaciones laborales, los inmigrantes tienen muchas menos probabilidades de trabajar indefinidos a tiempo completo, sobretodo en el caso de los hombres y más probabilidades de terminar sin trabajar ni cobrar prestación por desempleo. Finalmente los hombres emigrantes tienen más probabilidades que los nacionales de terminar el muestreo cobrando una prestación por desempleo pero las mujeres emigrantes tienen menos probabilidades que las nacionales de terminar en dicha situación.

Además, en los modelos especificados se ha controlado por otras variables relacionadas con el contexto económico y social del individuo como trabajar en una provincia distinta a aquella en la que se nació, vivir en una Comunidad Autónoma con un PIB per capita superior a la media española y vivir en una población de más de 40000 habitantes. Vivir en una Comunidad Autónoma con un PIB per capita superior a la media española disminuye las probabilidades de que un hombre trabaje a tiempo parcial pero no tiene ningún efecto en las mujeres. Vivir en una población de más de 40000 habitantes reduce las probabilidades de trabajar a TP de forma indefinida y aumenta ligeramente las de hacerlo de forma temporal. Trabajar en una provincia distinta a aquella en la que se nació también reduce las probabilidades de trabajar a TP de forma indefinida y aumenta las de trabajar a TP temporal entre los hombres.

\section{Conclusiones}

En general, en el trabajo a tiempo parcial del mercado laboral español predominan los trabajadores más vulnerables y con menos opciones: mujeres, trabajadores con niveles de cualificación bajos y, según que tipos de trabajo a tiempo parcial, inmigrantes. Esto hace pensar que, en una buena parte de los casos, no existe voluntariedad por parte del trabajador en la decisión de trabajar a tiempo parcial y que se trabaja a tiempo parcial porque el mercado, o las circunstancias personales, no les da otras opciones. De hecho, así lo indica buena parte de la literatura. El análisis realizado permite obtener algunas conclusiones que, por un lado matizan y, por otro, refuerzan esta afirmación. 
Un aspecto fundamental es que los determinantes del trabajo a tiempo parcial masculino casi nunca coinciden con los del TP femenino. Por un lado, el trabajo a TP es propio de hombres, y de mujeres aunque en mucha menor medida, al principio y al final de su carrera. Por otro, es propio de mujeres en edades intermedias, cuando es más común estar al cuidado de los hijos. Por tanto, la edad determina de maneras muy diferentes las situaciones laborales de ambos sexos en general y el trabajar o no a tiempo parcial en particular.

El número de contratos que se ha firmado a lo largo de la vida laboral como determinantes del trabajo a tiempo parcial también afecta de manera muy diferente a hombres y mujeres pero de manera muy parecida a ambos sexos cuando se utiliza como variable explicativa de trabajar a tiempo completo.

Las experiencias anteriores afectan a la situación laboral actual de ambos sexos, a veces de maneras similares y otras de maneras diferentes:

- Ambos sexos están sujetos al atrapamiento en la temporalidad a TC y, sin embargo, para ambos sexos haber trabajado a TP temporalmente influye positivamente en poder trabajar a TP de manera indefinida.

- También ambos sexos tienden a compaginar trabajo autónomo con trabajo a tiempo parcial pero de manera diferente: en el caso de los hombres trabajo a TP indefinido y, en el de las mujeres, trabajo a TP temporal.

- Como se ha señalado en varias ocasiones, el haber trabajado a TC de manera indefinida influye de manera diferente en las probabilidades que cada sexo tiene de trabajar a TP con carácter indefinido: Es mucho menos probable que un hombre que ha trabajado fijo a TC trabaje a TP indefinido que que lo haga una mujer. Detrás de este resultado se encuentran las interrupciones de las carreras laborales femeninas a edades intermedias por motivos de vida familiar.

- Por otra parte, el haber trabajado a TC de manera indefinida también influye de manera diferente en las probabilidades que cada sexo tiene de trabajar a TP con carácter temporal pero en este caso al revés: Es muy poco probable que una mujer que ha trabajado fija a TC trabaje a TP con un contrato temporal pero aumenta las probabilidades de que lo haga un hombre. Detrás de este resultado se encuentran los contratos temporales a TP previos a la jubilación que son más comunes, como se ha visto, entre los hombres.

Algunas situaciones, como haber experimentado desempleo y/o inactividad en los últimos cinco años son más comunes entre las mujeres (un 23\% de la mujeres frente a un $14 \%$ de la mujeres han tenido una experiencia de este tipo) pero afectan de manera 
parecida a hombres y mujeres. Otras variables como el último sector de ocupación o haber cambiado de sector a lo largo de la vida laboral también diferencian a hombres y mujeres y, no solamente a sus probabilidades de trabajar a tiempo parcial, sino a sus situaciones laborales en general.

En general, los resultados obtenidos sustentan la idea de que, si bien el trabajo a tiempo parcial es propio de trabajadores con reducidas opciones laborales, tiene una naturaleza muy distinta entre los hombres y entre las mujeres.

En lo que se refiere a las líneas de investigación futura podemos distinguir dos líneas. Por un lado investigaciones que, utilizando la misma base de datos que hemos utilizado aquí, es decir, la MCVL y sus sucesivas entregas anuales cuyas posibilidades siguen siendo inmensas sigan las siguientes vías. En principio, observar si el mercado de trabajo evoluciona de manera que las diferencias entre hombres y mujeres van desapareciendo o agrandándose. Más allá de repetir este análisis para sucesivas entregas de la MCVL, se abren algunas opciones. El siguiente paso puede ser definir las variables dependientes de manera que contengan información, no solo sobre la situación actual sino sobre la estabilidad en cada situación atendiendo al tiempo que se lleva en un puesto de trabajo y/o a los ingresos que implica. Por otro lado, el estudio del trabajo a tiempo parcial precisa de la disponibilidad de información, no solo de la vida laboral, sino de la vida familiar. Esta línea de investigación puede abordarse solo parcialmente utilizando la MCVL que no dispone de información precisa sobre variables como el estado civil o el número de hijos.

\section{REFERENCIAS BIBLIOGRÁFICAS}

Buddelmeyer H.; Mourre G. y Ward-Warmedinger, M. (2005), «Part-Time Work in EU Countries: Labour Market Mobility, Entry and Exit», IZA Discussion Paper 1550, Institute for the Study of Labor, Bonn.

Cebrián, I. y Toharia, L. (2007), La temporalidad en el empleo: atrapamiento y trayectorias, Ministerio de Trabajo y Asuntos Sociales, Madrid.

CES (1996), Informe sobre el trabajo a tiempo parcial, Informe 4/96, Consejo Económico y Social, Madrid.

Durán A. y SEVIlla, M. A. (2005), Una muestra continúa de vidas laborales, IEF, Madrid.

Muñoz de Bustillo Fernández E. y Antón, J. I. (2008), El Trabajo a Tiempo Parcial en España en el Contexto de la Unión Europea, Ministerio de Trabajo e Inmigración.

OECD (2002), Employment Outlook, París. Disponible en https://www.oecd.org/dataoecd/28/58/ 18960381.pdf 
Ramos Muñoz, B. (2007), «Comparación de las magnitudes estadísticas del Empleo según la Encuesta de Población Activa y la Muestra Continua de Vidas Laborales», Jornadas de Usuarios de la Muestra Continua de Vidas Laborales (octubre 2007).

Smith, M.; Cebrián, I.; Hernanz, V.; Malo, M. A. y Moreno, G. (2000), «Transitions in Spain and Great Britain: An Analysis with the Labour Force Survey», en J. O'Relly, I. Cebrian y M. Lallement (eds.), Working Time Changes: Social Integration through Time Transitional Labour Markets, Edward Elgar, Chentelham, United Kingdom, 173-204. 